\title{
On coronal structures and their variability in active stars: The case of Capella observed with Chandra/LETGS ${ }^{\star}$
}

\author{
C. Argiroffi ${ }^{1}$, A. Maggio $^{2}$, and G. Peres ${ }^{1}$ \\ 1 Dipartimento di Scienze Fisiche ed Astronomiche, Sezione di Astronomia, Università di Palermo, Piazza del Parlamento 1, \\ 90134 Palermo, Italy \\ 2 INAF - Osservatorio Astronomico di Palermo, Piazza del Parlamento 1, 90134 Palermo, Italy
}

Received 13 December 2002 / Accepted 27 March 2003

\begin{abstract}
In this paper we present a detailed analysis of two X-ray spectra of Capella, taken eleven months apart with the Low Energy Transmission Grating Spectrometer (LETGS) of the Chandra Observatory. We have studied variability of the coronal emission over different time scales, both in the whole X-ray band and in narrow temperature ranges identified by lines. The comparison of the two observations shows that the whole coronal emission of Capella in March 2000 was 3\% higher than in February 2001; there also appears to be a tendency, albeit a marginal one, for the hottest lines to show the largest changes between the two observations. A detailed search for short-term variability (on time scales ranging from $10^{2}$ to $10^{4} \mathrm{~s}$ ) in the emission of individual lines shows that in all cases the emission is compatible with a constant source; the firm upper limits of $5 \%-10 \%$ to the source variability on short time scales suggests that the intense X-ray emission is due to stable coronal structures and not to flaring activity. We have also determined the coronal thermal structure, as described with the emission measure distribution vs. temperature and with the help of plasma density, derived from the analysis of the O VII, Ne IX, Mg XI and Si XIII He-like ion triplets. The emission measure distribution, em $(T)$, and the element abundances, have been reconstructed with the Markov-Chain Monte Carlo method by Kashyap \& Drake; the $\operatorname{em}(T)$ presents a previously known sharp peak around $\log T=6.8-6.9$, but we have also found evidence of a small amount of plasma at $T>10^{7} \mathrm{~K}$. With the help of the $e m(T)$ and the density values we have estimated the pressure and volume of the emitting plasma at different temperatures, and we have derived information about the structure of individual loops and about the population of loops having different maximum temperatures. Our results indicate that loops with higher maximum temperature have higher pressure and smaller volume than lower temperature loops.
\end{abstract}

Key words. X-rays: stars - techniques: spectroscopic - stars: activity - stars: coronae - stars: individual: Capella

\section{Introduction}

It is known that many stars have X-ray coronal emission which is up to several orders of magnitude higher than the solar one. The knowledge of the solar corona is the starting point for studies of the stellar X-ray emission because of the high spatial resolution and high signal to noise ratio achievable in solar observations. Therefore it is assumed that also stellar coronal emission is produced by hot and optically thin plasma located in the outer atmospheres of the stars and confined in loop structures by magnetic field. However, when describing coronae which have emission up to $10^{4}$ times the solar value, we should exercise some care because we need to scale the solar corona to match the observed emission, and there are different ways to do this, for instance assuming larger emitting volumes and/or higher densities. Therefore, a natural question is which are the differences and which the analogies between

Send offprint requests to: C. Argiroffi, e-mail: argi@oapa.astropa.unipa.it

* Appendices A and B are only available in electronic form at http://www. edpsciences.org the structures characterizing the coronae of active stars and the solar one.

The high resolution X-ray spectra today available from Chandra and XMM-Newton observations allow us to improve our modeling of the structures present in active stellar coronae. With these spectra it is possible to evaluate fluxes of individual lines in the X-ray band, and hence to discriminate the emission from coronal regions at different temperatures, to reconstruct the emission measure distribution, $\operatorname{em}(T)$, and to estimate element abundances. Moreover electron density $N_{\mathrm{e}}$ and temperature $T$ of different coronal regions can be inferred using spectroscopic diagnostics based on line ratios, and starting from these parameters we can infer the pressure and volume of the emitting plasma. Finally, by combining all these results we can derive useful information about the coronal loop population.

In order to understand whether the coronal structures are steady or dynamic it is also crucial to test the presence of variability in the $\mathrm{X}$-ray emission over different time scales. The combined spectral and temporal resolutions in each observation allow us to perform variability studies on short time scales 
(minutes, hours) of the plasma in narrow temperature ranges by selecting and analyzing photon arrival times of individual emission lines. The advantage of this procedure is that we study the plasma in temperature ranges narrower than those explored with broad band analysis.

Finally, we can analyze long term variability (months, years), possibly linked to phenomena like stellar magnetic cycles, by comparing observations taken at different times.

In the light of the above considerations we have chosen to analyze the X-ray emission from Capella, because it is an active binary star whose spectra have high signal to noise ratio; furthermore its emission has never shown evident flares, and our observations confirm this behavior, with the additional bonus of allowing us to study its corona as largely composed of steady loops.

Capella is a close spectroscopic binary, at a distance of $12.93 \mathrm{pc}$, consisting of a G8 and a G1 giant stars, with masses of $2.7 M_{\odot}$ and $2.6 M_{\odot}$, and radii of $12 R_{\odot}$ and $9 R_{\odot}$, respectively. It is a member of the class of the long-period RS CVn binaries, having an orbital period of 104 days, not synchronous with the rotational period of the components, whose separation is $160 R_{\odot}$. The rotational velocities of the two components are quite different, in fact the G1 star rotates at $36 \mathrm{~km} \mathrm{~s}^{-1}$, much larger than the $3 \mathrm{~km} \mathrm{~s}^{-1}$ of the G8 star (Hummel et al. 1994).

Capella is a very bright X-ray source and for this reason it has been observed by every X-ray and EUV space-borne observatory. With the currently available instrumentation it is possible to separate the spectral contribution of each stellar component in the UV band, but not in the X-ray band. Linsky et al. (1998) have analyzed spectra taken in 1995 and in 1996 with the Goddard High-Resolution Spectrometer of the Hubble Space Telescope (HST), and, by deblending the profile of the Fe XXI line at $1354 \AA$ (which forms at $10^{7} \mathrm{~K}$ ), they found similar contributions from the two stellar components. Johnson et al. (2002), instead, analyzing the same Fe XXI line in the spectrum taken in 1999 with the HST/Space Telescope Imaging Spectrograph, have found that the G8 star contribution is negligible compared to the rapidly rotating G1 star. Johnson et al. suggest that the coronal emission of the G8 star is variable over time scales of a few years. This evidence is compatible with the results found by Dupree et al. (1999) who have analyzed several spectra obtained with EUVE since 1992; they have found variations, over time scales of about one year, of the coronal emission and of the hot part of the emission measure distribution. However a puzzling question is posed by the results reported by Young et al. (2001): they have analyzed a spectrum obtained with the Far Ultraviolet Spectroscopic Explorer in 2000, and they have found that the Fe XVIII line at $974.85 \AA$, which forms at $T=10^{6.8} \mathrm{~K}$, mainly comes from the $\mathrm{G} 8 \mathrm{com}-$ ponent.

Several authors, based on data from EUVE, (Dupree et al. 1993; Schrijver et al. 1995; Dupree et al. 1999; Brickhouse et al. 2000) have reconstructed the emission measure distribution, showing that the corona of Capella is characterized by a continuous temperature distribution with a sharp peak at $\log T \sim$ 6.8. Recent works (Brinkman et al. 2000; Canizares et al. 2000; Phillips et al. 2001; Ness et al. 2001; Mewe et al. 2001; Behar et al. 2001; Audard et al. 2001) report the results obtained from the analysis of the high resolution spectra of Capella gathered with Chandra and XMM-Newton. They have used individual line fluxes to perform density and temperature diagnostics, to infer some indication about the temperature distribution, and to derive some properties about the loop structures, but none of these works presents a detailed differential emission measure reconstruction.

In this paper we report the analysis of two X-ray spectra of Capella obtained with the LETGS of the Chandra Observatory. Our aim, as stated above, is to evaluate the emission measure distribution and to analyze the He-like ion triplets to infer some properties about the population of loops in the corona of Capella. We have also investigated in detail the presence of any short-term variability, checking the X-ray light curves of individual emission lines. Moreover we have compared the results found from the two spectra analyzed, to evaluate any variation occurred in one year.

In Sect. 2 we report the data processing and the methods used for the analysis. The results are shown in Sect. 3, while in Sect. 4 we discuss our results and report our conclusions in Sect. 5.

\section{Observations and data analysis}

The LETGS on the Chandra X-ray observatory is a diffraction grating spectrometer which provides spectra in the wavelength range $1-170 \AA$, with nominal resolution of $0.05 \AA$ (FWHM) over the whole bandpass.

We have analyzed two observations of Capella obtained with the LETGS on March 8, 2000, and on February 14, 2001, retrieved from the Chandra $X$-ray Center public archive. The exposure times are respectively $33.9 \mathrm{ks}$ and $26.9 \mathrm{ks}$. These observations are part of the on-going long-term calibration of the Chandra spectrometers and, among those available in the Chandra public archive, they have a similar and relatively long exposure time. This characteristic allows us to compare easily the results obtained from the analysis of the two spectra, and to study possible variations in the corona of Capella over time scales of about one year. Moreover, each observation is sufficiently long to allow us to study short-term variability on time scales from few minutes to few hours.

The data have been re-processed using the standard tools in the software package CIAO V2.1. The end products of this processing are positive and negative-order source spectra extracted from bow tie regions which ensure an almost constant fraction (90\%-95\%, depending on the wavelength) of source photons collected at each wavelength. Background spectra are also extracted from two regions above and below the source spectrum, each one having a constant area scaling factor of 5 with respect to the source region.

We have performed the analysis of the source spectra using the software package PINTofALE V1.0 developed by Kashyap \& Drake (2000) while the variability analysis within each observation was performed with the Collura et al. (1987) method (Sect. 3.5). The spectral analysis consisted of the following steps: line identification and fitting, reconstruction of the emission measure distribution with simultaneous determination of the element abundances, density and temperature diagnostics 

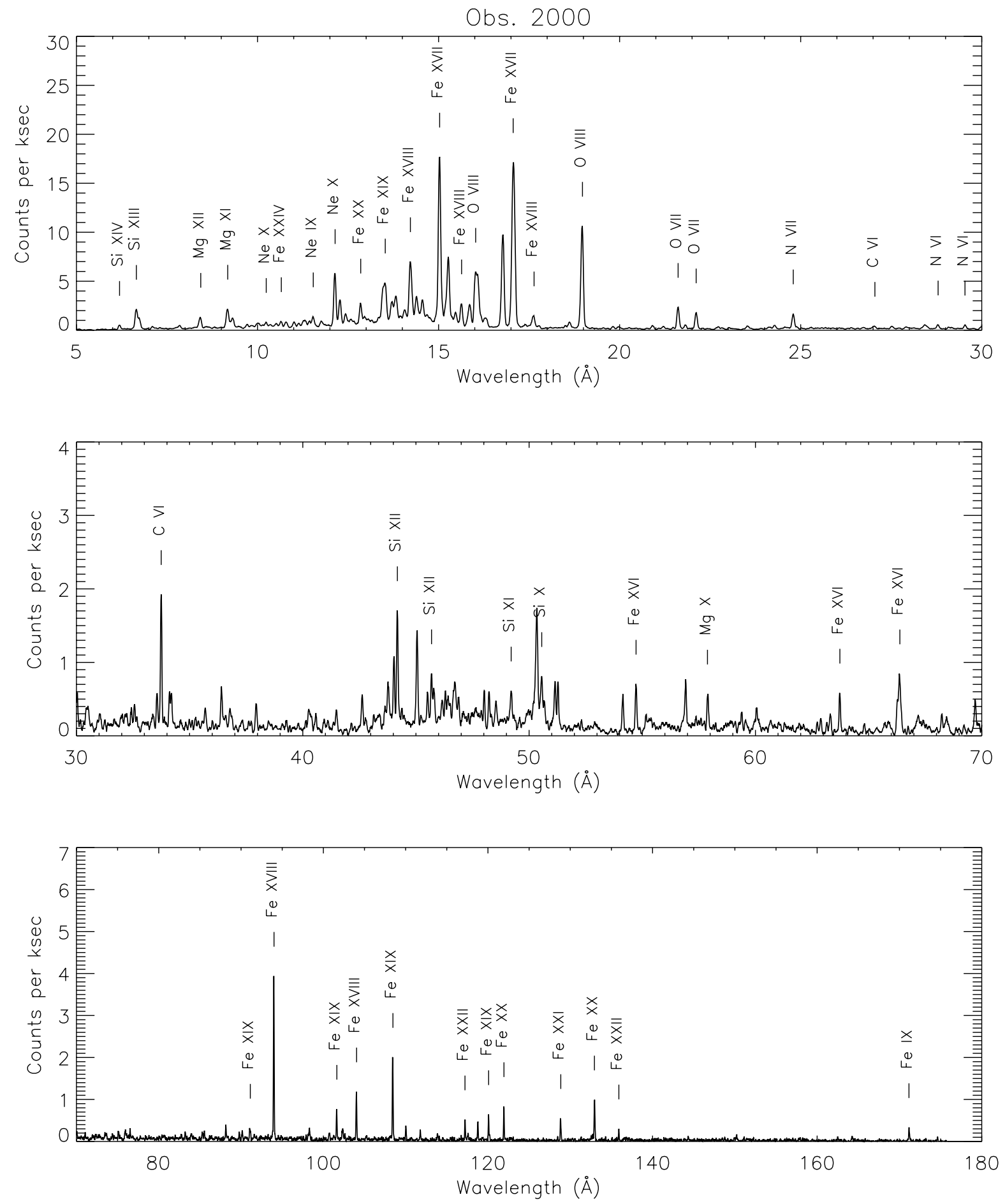

Fig. 1. LETGS smoothed spectrum (positive and negative orders added) of Capella, with bin size of $0.0125 \AA$, collected on March 8, 2000, split into three parts. Note the different scales in each panel. The labels identify some of the strongest lines.

using He-like triplets. For the above analysis we have adopted the CHIANTI V3.03 emission line database, and the Mazzotta et al. (1998) ionization equilibrium.

In each spectrum we have identified and measured the fluxes of the strongest emission lines which are listed in Table 1. As an example, the spectrum of Capella observed in 2000 is shown in Fig. 1. The spectrum extracted from the 2001 observation is virtually identical.
In order to perform the line fitting we have assumed, for the line profile, a beta-model of the form:

$I(\lambda)=I_{\max } \frac{1}{\left[1+\left(\frac{\lambda-\lambda_{\mathrm{c}}}{\Delta \lambda}\right)^{2}\right]^{\beta}}$

with the parameters $\Delta \lambda=0.03 \AA$ and $\beta=1.8 \mathrm{kept}$ fixed, while the values of $\lambda_{\mathrm{c}}$ and of the total line counts have been derived with the fitting procedure. The continuum has been 
Table 1. Strongest lines in the Capella spectrum.

\begin{tabular}{|c|c|c|c|c|c|c|c|c|c|}
\hline \multirow[b]{2}{*}{ Label } & \multirow[b]{2}{*}{$\lambda_{\mathrm{obs}+}^{a}$} & \multirow[b]{2}{*}{$\lambda_{\mathrm{obs}-}^{a}$} & \multicolumn{3}{|r|}{ Identification } & \multirow[b]{2}{*}{$\log T_{\max }^{b}$} & \multirow{2}{*}{$\begin{array}{c}2000 \\
(F \pm \sigma)^{c}\end{array}$} & 2001 & \multirow[b]{2}{*}{$e m(T)^{d}$} \\
\hline & & & $\lambda_{\text {pred }}^{a}$ & Ion & Transition ( & & & $(F \pm \sigma)^{c}$ & \\
\hline 1a & 6.19 & 6.19 & 6.18 & Si XIV & $2 \mathrm{p}^{2} \mathrm{P}_{3 / 2} \rightarrow 1 \mathrm{~s}^{2} \mathrm{~S}_{1 / 2}$ & 7.20 & $105 \pm 15$ & $66 \pm 13$ & $\mathrm{AB}$ \\
\hline $1 b$ & .. & .. & 6.19 & Si XIV & $2 \mathrm{p}^{2} \mathrm{P}_{1 / 2} \rightarrow 1 \mathrm{~s}^{2} \mathrm{~S}_{1 / 2}$ & 7.20 & .. & .. & \\
\hline 2 & 6.65 & 6.64 & 6.65 & Si XIII & $1 \mathrm{~s} 2 \mathrm{p}^{1} \mathrm{P}_{1} \rightarrow 1 \mathrm{~s}^{2}{ }^{1} \mathrm{~S}_{0}$ & 7.00 & $417 \pm 26$ & $297 \pm 23$ & $\mathrm{AB}$ \\
\hline 3 & 6.70 & 6.69 & 6.69 & Si XIII & $1 \mathrm{~s} 2 \mathrm{p}^{3} \mathrm{P}_{1} \rightarrow 1 \mathrm{~s}^{2}{ }^{1} \mathrm{~S}_{0}$ & 6.95 & $99 \pm 20$ & $57 \pm 18$ & \\
\hline 4 & 6.75 & 6.73 & 6.74 & Si XIII & $1 \mathrm{~s} 2 \mathrm{~s}^{3} \mathrm{~S}_{1} \rightarrow 1 \mathrm{~s}^{2}{ }^{1} \mathrm{~S}_{0}$ & 7.00 & $202 \pm 20$ & $174 \pm 19$ & \\
\hline $5 \mathrm{a}$ & 8.43 & 8.40 & 8.42 & Mg XII & $2 \mathrm{p}^{2} \mathrm{P}_{3 / 2} \rightarrow 1 \mathrm{~s}^{2} \mathrm{~S}_{1 / 2}$ & 7.00 & $297 \pm 22$ & $230 \pm 20$ & $\mathrm{AB}$ \\
\hline $5 b$ & .. & .. & 8.42 & Mg XII & $2 \mathrm{p}^{2} \mathrm{P}_{1 / 2} \rightarrow 1 \mathrm{~s}^{2} \mathrm{~S}_{1 / 2}$ & 7.00 & .. & $\ldots$ & \\
\hline 6 & 9.18 & 9.16 & 9.17 & Mg XI & $1 \mathrm{~s} 2 \mathrm{p}^{1} \mathrm{P}_{1} \rightarrow 1 \mathrm{~s}^{2}{ }^{1} \mathrm{~S}_{0}$ & 6.80 & $446 \pm 26$ & $348 \pm 24$ & $\mathrm{AB}$ \\
\hline 7 & 9.26 & 9.22 & 9.23 & $\mathrm{Mg} \mathrm{XI}$ & $1 \mathrm{~s} 2 \mathrm{p}^{3} \mathrm{P}_{1} \rightarrow 1 \mathrm{~s}^{2}{ }^{1} \mathrm{~S}_{0}$ & 6.80 & $110 \pm 18$ & $88 \pm 16$ & \\
\hline 8 & 9.32 & 9.30 & 9.31 & Mg XI & $1 \mathrm{~s} 2 \mathrm{~s}^{3} \mathrm{~S}_{1} \rightarrow 1 \mathrm{~s}^{2}{ }^{1} \mathrm{~S}_{0}$ & 6.80 & $236 \pm 21$ & $209 \pm 19$ & \\
\hline $9 \mathrm{a}$ & 10.24 & 10.23 & 10.24 & $\mathrm{Ne} \mathrm{X}$ & $3 \mathrm{p}^{2} \mathrm{P}_{3 / 2} \rightarrow 1 \mathrm{~s}^{2} \mathrm{~S}_{1 / 2}$ & 6.80 & $108 \pm 18$ & $96 \pm 17$ & \\
\hline $9 b$ & .. & .. & 10.24 & $\mathrm{Ne} \mathrm{x}$ & $3 \mathrm{p}^{2} \mathrm{P}_{1 / 2} \rightarrow 1 \mathrm{~s}^{2} \mathrm{~S}_{1 / 2}$ & 6.80 & $\ldots$ & .. & \\
\hline $10 \mathrm{a}$ & 10.66 & 10.65 & 10.62 & Fe XXIV & $1 \mathrm{~s}^{2} 3 \mathrm{p}^{2} \mathrm{P}_{3 / 2} \rightarrow 1 \mathrm{~s}^{2} 2 \mathrm{~s}^{2} \mathrm{~S}_{1 / 2}$ & 7.30 & $126 \pm 18$ & $77 \pm 16$ & $\mathrm{AB}$ \\
\hline $10 \mathrm{~b}$ & .. & .. & 10.66 & Fe XXIV & $1 s^{2} 3 p^{2} \mathrm{P}_{1 / 2} \rightarrow 1 s^{2} 2 s^{2} \mathrm{~S}_{1 / 2}$ & 7.30 & $\ldots$ & .. & \\
\hline $11 \mathrm{a}$ & 11.02 & 11.00 & 10.98 & Fe XXIII & $2 \mathrm{~s} 3 \mathrm{p}{ }^{1} \mathrm{P}_{1} \rightarrow 2 \mathrm{~s}^{2}{ }^{1} \mathrm{~S}_{0}$ & 7.20 & $114 \pm 18$ & $75 \pm 16$ & $\mathrm{AB}$ \\
\hline $11 \mathrm{~b}$ & $\cdots$ & $\cdots$ & 11.02 & Fe XXIII & $2 \mathrm{~s} 3 \mathrm{p}^{3} \mathrm{P}_{1} \rightarrow 2 \mathrm{~s}^{2}{ }^{1} \mathrm{~S}_{0}$ & 7.20 & $\cdots$ & $\cdots$ & \\
\hline $11 \mathrm{c}$ & $\cdots$ & $\cdots$ & 11.02 & $\mathrm{Fe}$ XVII & $2 \mathrm{~s} 2 \mathrm{p}^{6} 4 \mathrm{p}^{1} \mathrm{P}_{1} \rightarrow 2 \mathrm{p}^{6}{ }^{1} \mathrm{~S}_{0}$ & 6.80 & $\cdots$ & $\cdots$ & \\
\hline $11 d$ & $\cdots$ & .. & 11.03 & Fe XXIV & $1 \mathrm{~s}^{2} 3 \mathrm{~d}^{2} \mathrm{D}_{3 / 2} \rightarrow 1 \mathrm{~s}^{2} 2 \mathrm{p}^{2} \mathrm{P}_{1 / 2}$ & 7.30 & .. & .. & \\
\hline 12 & 11.14 & 11.13 & 11.17 & Fe XXIV & $1 \mathrm{~s}^{2} 3 \mathrm{~d}^{2} \mathrm{D}_{5 / 2} \rightarrow 1 \mathrm{~s}^{2} 2 \mathrm{p}^{2} \mathrm{P}_{3 / 2}$ & 7.30 & $84 \pm 17$ & $60 \pm 15$ & $\mathrm{AB}$ \\
\hline $13 \mathrm{a}$ & 11.44 & 11.42 & 11.43 & Fe XXIV & $1 \mathrm{~s}^{2} 3 \mathrm{~s}^{2} \mathrm{~S}_{1 / 2} \rightarrow 1 \mathrm{~s}^{2} 2 \mathrm{p}^{2} \mathrm{P}_{3 / 2}$ & 7.30 & $133 \pm 19$ & $102 \pm 17$ & $\mathrm{AB}$ \\
\hline $13 b$ & $\cdots$ & $\cdots$ & 11.44 & Fe XXII & $2 \mathrm{~s} 2 \mathrm{p}\left({ }^{3} \mathrm{P}\right) 3 \mathrm{p}{ }^{2} \mathrm{D}_{3 / 2} \rightarrow 2 \mathrm{~s}^{2} 2 \mathrm{p}^{2} \mathrm{P}_{1 / 2}$ & 7.10 & $\cdots$ & $\cdots$ & \\
\hline 14 & 11.54 & 11.53 & 11.55 & $\mathrm{Ne}$ IX & $1 \mathrm{~s} 3 \mathrm{p}^{1} \mathrm{P}_{1} \rightarrow 1 \mathrm{~s}^{2}{ }^{1} \mathrm{~S}_{0}$ & 6.60 & $243 \pm 22$ & $176 \pm 19$ & \\
\hline $15 \mathrm{a}$ & 11.78 & 11.75 & 11.74 & Fe XXIII & $2 \mathrm{~s} 3 \mathrm{~d}^{1} \mathrm{D}_{2} \rightarrow 2 \mathrm{~s} 2 \mathrm{p}^{1} \mathrm{P}_{1}$ & 7.20 & $149 \pm 19$ & $158 \pm 19$ & $\mathrm{AB}$ \\
\hline $15 b$ & $\cdots$ & $\cdots$ & 11.77 & Fe XXII & $2 s^{2}\left({ }^{1} S\right) 3 d^{2} D_{3 / 2} \rightarrow 2 s^{2} 2 p^{2} P_{1 / 2}$ & 7.10 & $\cdots$ & $\cdots$ & \\
\hline $16 \mathrm{a}$ & 12.14 & 12.13 & 12.12 & Fe XVII & $2 \mathrm{p}^{5} 4 \mathrm{~d}^{1} \mathrm{P}_{1} \rightarrow 2 \mathrm{p}^{6}{ }^{1} \mathrm{~S}_{0}$ & 6.80 & $1139 \pm 40$ & $899 \pm 36$ & $\mathrm{AB}$ \\
\hline $16 \mathrm{~b}$ & $\cdots$ & $\cdots$ & 12.13 & $\mathrm{Ne} \mathrm{X}$ & $2 \mathrm{p}^{2} \mathrm{P}_{3 / 2} \rightarrow 1 \mathrm{~s}^{2} \mathrm{~S}_{1 / 2}$ & 6.80 & $\cdots$ & $\cdots$ & \\
\hline $16 \mathrm{c}$ & $\ldots$ & $\ldots$ & 12.14 & $\mathrm{Ne} \mathbf{x}$ & $2 \mathrm{p}^{2} \mathrm{P}_{1 / 2} \rightarrow 1 \mathrm{~s}^{2} \mathrm{~S}_{1 / 2}$ & 6.80 & $\ldots$ & $\ldots$ & \\
\hline $17 \mathrm{a}$ & 12.28 & 12.27 & 12.26 & Fe XVII & $2 \mathrm{p}^{5} 4 \mathrm{~d}^{3} \mathrm{D}_{1} \rightarrow 2 \mathrm{p}^{6}{ }^{1} \mathrm{~S}_{0}$ & 6.80 & $573 \pm 31$ & $349 \pm 25$ & $\mathrm{AB}$ \\
\hline $17 \mathrm{~b}$ & $\cdots$ & .. & 12.28 & Fe XXI & $2 \mathrm{~s}^{2} 2 \mathrm{p} 3 \mathrm{~d}^{3} \mathrm{D}_{1} \rightarrow 2 \mathrm{p}^{2}{ }^{3} \mathrm{P}_{0}$ & 7.00 & .. & $\ldots$ & \\
\hline 18 & 12.44 & 12.42 & 12.44 & Ni XIX & $2 \mathrm{p}^{5} 3 \mathrm{~d}^{1} \mathrm{P}_{1} \rightarrow 2 \mathrm{p}^{6}{ }^{1} \mathrm{~S}_{0}$ & 6.90 & $275 \pm 24$ & $214 \pm 21$ & $\mathrm{AB}$ \\
\hline $19 \mathrm{a}$ & 12.85 & 12.84 & 12.82 & $\mathrm{Fe} \mathrm{XX}$ & $2 \mathrm{~s}^{2} 2 \mathrm{p}^{2}\left({ }^{3} \mathrm{P}\right) 3 \mathrm{~d}^{4} \mathrm{P}_{3 / 2} \rightarrow 2 \mathrm{~s}^{2} 2 \mathrm{p}^{3}{ }^{4} \mathrm{~S}_{3 / 2}$ & 7.00 & $491 \pm 29$ & $338 \pm 24$ & $\mathrm{AB}$ \\
\hline $19 b$ & $\cdots$ & $\cdots$ & 12.82 & Fe $\mathrm{xx}$ & $2 \mathrm{~s}^{2} 2 \mathrm{p}^{2}\left({ }^{3} \mathrm{P}\right) 3 \mathrm{~d}^{4} \mathrm{P}_{5 / 2} \rightarrow 2 \mathrm{~s}^{2} 2 \mathrm{p}^{3}{ }^{4} \mathrm{~S}_{3 / 2}$ & 7.00 & $\cdots$ & $\cdots$ & \\
\hline $20 \mathrm{a}$ & 13.46 & 13.45 & 13.45 & $\mathrm{Ne}$ IX & $1 \mathrm{~s} 2 \mathrm{p}^{1} \mathrm{P}_{1} \rightarrow 1 \mathrm{~s}^{2}{ }^{1} \mathrm{~S}_{0}$ & 6.60 & $730 \pm 35$ & $619 \pm 32$ & \\
\hline $20 \mathrm{~b}$ & .. & $\cdots$ & 13.46 & Fe XIX & $2 \mathrm{p}^{3}\left({ }^{2} \mathrm{D}^{*}\right) 3 \mathrm{~d}^{3} \mathrm{~S}_{1} \rightarrow 2 \mathrm{~s}^{2} 2 \mathrm{p}^{4}{ }^{3} \mathrm{P}_{2}$ & 6.90 & .. & $\cdots$ & \\
\hline 21 & 13.53 & 13.51 & 13.52 & Fe XIX & $2 \mathrm{p}^{3}\left({ }^{2} \mathrm{D}^{*}\right) 3 \mathrm{~d}^{3} \mathrm{D}_{3} \rightarrow 2 \mathrm{~s}^{2} 2 \mathrm{p}^{4}{ }^{3} \mathrm{P}_{2}$ & 6.90 & $723 \pm 37$ & $552 \pm 33$ & $\mathrm{AB}$ \\
\hline $22 \mathrm{a}$ & 13.57 & 13.55 & 13.55 & $\mathrm{Ne}$ IX & $1 \mathrm{~s} 2 \mathrm{p}{ }^{3} \mathrm{P}_{1} \rightarrow 1 \mathrm{~s}^{2}{ }^{1} \mathrm{~S}_{0}$ & 6.55 & $330 \pm 30$ & $265 \pm 28$ & \\
\hline $22 b$ & $\cdots$ & $\cdots$ & 13.57 & Fe XIX & $2 \mathrm{p}^{3}\left({ }^{2} \mathrm{D}^{*}\right) 3 \mathrm{~d}^{3} \mathrm{P}_{2} \rightarrow 2 \mathrm{~s}^{2} 2 \mathrm{p}^{4}{ }^{3} \mathrm{P}_{2}$ & 6.90 & $\cdots$ & $\cdots$ & \\
\hline 23 & 13.66 & 13.65 & 13.67 & Fe XIX & $2 p^{3}\left({ }^{2} D^{*}\right) 3 d^{3} D_{2} \rightarrow 2 s^{2} 2 p^{4}{ }^{3} P_{1}$ & 6.90 & $143 \pm 22$ & $144 \pm 21$ & A \\
\hline 24 & 13.71 & 13.70 & 13.70 & $\mathrm{Ne}$ IX & $1 \mathrm{~s} 2 \mathrm{~s}^{3} \mathrm{~S}_{1} \rightarrow 1 \mathrm{~s}^{2}{ }^{1} \mathrm{~S}_{0}$ & 6.55 & $402 \pm 30$ & $306 \pm 26$ & \\
\hline $25 \mathrm{a}$ & 13.76 & 13.73 & 13.74 & Fe XIX & $2 \mathrm{p}^{3}\left({ }^{2} \mathrm{D}^{*}\right) 3 \mathrm{~d}^{1} \mathrm{~F}_{3} \rightarrow 2 \mathrm{~s}^{2} 2 \mathrm{p}^{4}{ }^{1} \mathrm{D}_{2}$ & 6.90 & $178 \pm 27$ & $161 \pm 24$ & \\
\hline $25 b$ & $\cdots$ & .. & 13.74 & Fe $\mathrm{xx}$ & $2 s^{2} 2 p^{2}\left({ }^{3} P\right) 3 s^{4} P_{5 / 2} \rightarrow 2 s^{2} 2 p^{3}{ }^{4} S_{3 / 2}$ & 7.00 & $\cdots$ & .. & \\
\hline $26 \mathrm{a}$ & 13.81 & 13.78 & 13.78 & Ni XIX & $2 \mathrm{p}^{5} 3 \mathrm{~s}{ }^{1} \mathrm{P}_{1} \rightarrow 2 \mathrm{p}^{6}{ }^{1} \mathrm{~S}_{0}$ & 6.80 & $387 \pm 31$ & $244 \pm 27$ & $\mathrm{AB}$ \\
\hline $26 b$ & $\cdots$ & $\cdots$ & 13.80 & Fe XIX & $2 \mathrm{p}^{3}\left({ }^{4} \mathrm{~S}^{*}\right) 3 \mathrm{~d}^{3} \mathrm{D}_{3} \rightarrow 2 \mathrm{~s}^{2} 2 \mathrm{p}^{4}{ }^{3} \mathrm{P}_{2}$ & 6.90 & $\cdots$ & $\cdots$ & \\
\hline 27 & 13.84 & 13.83 & 13.82 & Fe XVII & $2 \mathrm{~s} 2 \mathrm{p}^{6} 3 \mathrm{p}{ }^{1} \mathrm{P}_{1} \rightarrow 2 \mathrm{p}^{6}{ }^{1} \mathrm{~S}_{0}$ & 6.80 & $400 \pm 31$ & $257 \pm 27$ & $\mathrm{AB}$ \\
\hline 28 & 13.90 & 13.86 & 13.89 & Fe XVII & $2 \mathrm{~s} 2 \mathrm{p}^{6} 3 \mathrm{p}^{3} \mathrm{P}_{1} \rightarrow 2 \mathrm{p}^{6}{ }^{1} \mathrm{~S}_{0}$ & 6.75 & $134 \pm 23$ & $99 \pm 20$ & \\
\hline $29 \mathrm{a}$ & 13.96 & 13.94 & 13.95 & $\mathrm{Fe} \mathrm{xx}$ & $2 s^{2} 2 p^{2}\left({ }^{3} P\right) 3 s^{4} P_{1 / 2} \rightarrow 2 s^{2} 2 p^{3}{ }^{4} S_{3 / 2}$ & 7.00 & $210 \pm 22$ & $133 \pm 19$ & $\mathrm{AB}$ \\
\hline $29 \mathrm{~b}$ & ... & .. & 13.95 & Fe XVIII & $2 \mathrm{p}^{4}\left({ }^{1} \mathrm{~S}\right) 3 \mathrm{~d}^{2} \mathrm{D}_{5 / 2} \rightarrow 2 \mathrm{~s}^{2} 2 \mathrm{p}^{5}{ }^{2} \mathrm{P}_{3 / 2}$ & 6.90 & .. & .. & \\
\hline 30 & 14.06 & 14.04 & 14.04 & Ni XIX & $2 \mathrm{p}^{5} 3 \mathrm{~s}^{3} \mathrm{P}_{1} \rightarrow 2 \mathrm{p}^{6}{ }^{1} \mathrm{~S}_{0}$ & 6.80 & $308 \pm 25$ & $258 \pm 23$ & $\mathrm{AB}$ \\
\hline 31 & 14.10 & 14.10 & 14.08 & Ni XIX & $2 \mathrm{p}^{5} 3 \mathrm{~s}^{3} \mathrm{P}_{2} \rightarrow 2 \mathrm{p}^{6}{ }^{1} \mathrm{~S}_{0}$ & 6.80 & $195 \pm 23$ & $147 \pm 20$ & $\mathrm{AB}$ \\
\hline $32 \mathrm{a}$ & 14.22 & 14.20 & 14.20 & Fe XVIII & $2 \mathrm{p}^{4}\left({ }^{1} \mathrm{D}\right) 3 \mathrm{~d}^{2} \mathrm{D}_{5 / 2} \rightarrow 2 \mathrm{~s}^{2} 2 \mathrm{p}^{5}{ }^{2} \mathrm{P}_{3 / 2}$ & 6.90 & $1318 \pm 44$ & $1044 \pm 39$ & \\
\hline $32 b$ & $\cdots$ & $\cdots$ & 14.21 & Fe XVIII & $2 \mathrm{p}^{4}\left({ }^{1} \mathrm{D}\right) 3 \mathrm{~d}^{2} \mathrm{P}_{3 / 2} \rightarrow 2 \mathrm{~s}^{2} 2 \mathrm{p}^{5}{ }^{2} \mathrm{P}_{3 / 2}$ & 6.90 & $\cdots$ & $\cdots$ & \\
\hline 33 & 14.27 & 14.26 & 14.26 & Fe XVIII & $2 \mathrm{p}^{4}\left({ }^{1} \mathrm{D}\right) 3 \mathrm{~d}^{2} \mathrm{~S}_{1 / 2} \rightarrow 2 \mathrm{~s}^{2} 2 \mathrm{p}^{5}{ }^{2} \mathrm{P}_{3 / 2}$ & 6.90 & $528 \pm 34$ & $313 \pm 28$ & $\mathrm{AB}$ \\
\hline
\end{tabular}


Table 1. continued.

\begin{tabular}{|c|c|c|c|c|c|c|c|c|c|c|}
\hline \multirow[b]{2}{*}{ Label } & \multirow[b]{2}{*}{$\lambda_{\mathrm{obs}+}^{a}$} & \multirow[b]{2}{*}{$\lambda_{\mathrm{obs}-}^{a}$} & \multicolumn{4}{|r|}{ Identification } & \multirow[b]{2}{*}{$\log T_{\max }^{b}$} & \multirow{2}{*}{$\frac{2000}{(F+\sigma)^{c}}$} & 2001 & \multirow[b]{2}{*}{$e m(T)^{d}$} \\
\hline & & & $\lambda_{\mathrm{pred}}^{a}$ & & Ion & Transition ( upper $\rightarrow$ lower $)$ & & & $(F \pm \sigma)^{c}$ & \\
\hline $34 \mathrm{a}$ & 14.39 & 14.39 & 14.36 & $\mathrm{Fe}$ & XVIII & $2 \mathrm{p}^{4}\left({ }^{1} \mathrm{D}\right) 3 \mathrm{~d}^{2} \mathrm{D}_{3 / 2} \rightarrow 2 \mathrm{~s}^{2} 2 \mathrm{p}^{5}{ }^{2} \mathrm{P}_{1 / 2}$ & 6.90 & $667 \pm 32$ & $498 \pm 28$ & $\mathrm{AB}$ \\
\hline $34 \mathrm{~b}$ & $\cdots$ & $\cdots$ & 14.37 & $\mathrm{Fe}$ & XVIII & $2 \mathrm{p}^{4}\left({ }^{3} \mathrm{P}\right) 3 \mathrm{~d}^{2} \mathrm{D}_{5 / 2} \rightarrow 2 \mathrm{~s}^{2} 2 \mathrm{p}^{5}{ }^{2} \mathrm{P}_{3 / 2}$ & 6.90 & .. & $\cdots$ & \\
\hline $35 \mathrm{a}$ & 14.56 & 14.56 & 14.53 & $\mathrm{Fe}$ & XVIII & $2 \mathrm{p}^{4}\left({ }^{3} \mathrm{P}\right) 3 \mathrm{~d}^{2} \mathrm{~F}_{5 / 2} \rightarrow 2 \mathrm{~s}^{2} 2 \mathrm{p}^{5}{ }^{2} \mathrm{P}_{3 / 2}$ & 6.90 & $629 \pm 31$ & $450 \pm 27$ & $\mathrm{AB}$ \\
\hline $35 b$ & .. & .. & 14.55 & $\mathrm{Fe}$ & XVIII & $2 \mathrm{p}^{4}\left({ }^{3} \mathrm{P}\right) 3 \mathrm{~d}^{4} \mathrm{P}_{3 / 2} \rightarrow 2 \mathrm{~s}^{2} 2 \mathrm{p}^{5}{ }^{2} \mathrm{P}_{3 / 2}$ & 6.90 & .. & .. & \\
\hline 36 & 15.03 & 15.02 & 15.02 & $\mathrm{Fe}$ & XVII & $2 \mathrm{p}^{5} 3 \mathrm{~d}^{1} \mathrm{P}_{1} \rightarrow 2 \mathrm{p}^{6}{ }^{1} \mathrm{~S}_{0}$ & 6.75 & $3892 \pm 65$ & $3014 \pm 59$ & $\mathrm{AB}$ \\
\hline $37 \mathrm{a}$ & 15.20 & 15.19 & 15.18 & $\mathrm{O}$ & VIII & $4 \mathrm{p}^{2} \mathrm{P}_{3 / 2} \rightarrow 1 \mathrm{~s}^{2} \mathrm{~S}_{1 / 2}$ & 6.50 & $433 \pm 30$ & $389 \pm 28$ & \\
\hline $37 \mathrm{~b}$ & $\cdots$ & $\cdots$ & 15.18 & $\mathrm{O}$ & VIII & $4 \mathrm{p}^{2} \mathrm{P}_{1 / 2} \rightarrow 1 \mathrm{~s}^{2} \mathrm{~S}_{1 / 2}$ & 6.50 & $\cdots$ & $\cdots$ & \\
\hline $37 \mathrm{c}$ & .. & .. & 15.20 & $\mathrm{Fe}$ & XIX & $2 \mathrm{p}^{3}\left({ }^{4} \mathrm{~S}^{*}\right) 3 \mathrm{~s}^{5} \mathrm{~S}_{2} \rightarrow 2 \mathrm{~s}^{2} 2 \mathrm{p}^{4}{ }^{3} \mathrm{P}_{2}$ & 6.90 & .. & $\ldots$ & \\
\hline 38 & 15.28 & 15.27 & 15.26 & $\mathrm{Fe}$ & XVII & $2 \mathrm{p}^{5} 3 \mathrm{~d}^{3} \mathrm{D}_{1} \rightarrow 2 \mathrm{p}^{6}{ }^{1} \mathrm{~S}_{0}$ & 6.75 & $1550 \pm 45$ & $1037 \pm 39$ & $\mathrm{AB}$ \\
\hline 39 & 15.45 & 15.45 & 15.45 & $\mathrm{Fe}$ & XVII & $2 \mathrm{p}^{5} 3 \mathrm{~d}^{3} \mathrm{P}_{1} \rightarrow 2 \mathrm{p}^{6}{ }^{1} \mathrm{~S}_{0}$ & 6.70 & $195 \pm 23$ & $169 \pm 21$ & $\mathrm{AB}$ \\
\hline 40 & 15.48 & 15.51 & 15.50 & $\mathrm{Fe}$ & XVIII & $2 \mathrm{~s} 2 \mathrm{p}^{5}\left({ }^{1} \mathrm{P}\right) 3 \mathrm{~s}^{2} \mathrm{P}_{3 / 2} \rightarrow 2 \mathrm{~s} 2 \mathrm{p}^{6}{ }^{2} \mathrm{~S}_{1 / 2}$ & 6.90 & $177 \pm 22$ & $112 \pm 19$ & $\mathrm{AB}$ \\
\hline $41 \mathrm{a}$ & 15.64 & 15.62 & 15.62 & $\mathrm{Fe}$ & XVIII & $2 \mathrm{p}^{4}\left({ }^{1} \mathrm{D}\right) 3 \mathrm{~s}{ }^{2} \mathrm{D}_{5 / 2} \rightarrow 2 \mathrm{~s}^{2} 2 \mathrm{p}^{5}{ }^{2} \mathrm{P}_{3 / 2}$ & 6.90 & $518 \pm 29$ & $351 \pm 25$ & \\
\hline $41 \mathrm{~b}$ & $\cdots$ & $\cdots$ & 15.63 & $\mathrm{Fe}$ & $\mathrm{XX}$ & $2 s^{2} 2 p^{2}\left({ }^{3} P\right) 3 p{ }^{4} S_{3 / 2} \rightarrow 2 s 2 p^{4}{ }^{2} S_{1 / 2}$ & 7.00 & .. & .. & \\
\hline 42 & 15.84 & 15.82 & 15.83 & $\mathrm{Fe}$ & XVIII & $2 \mathrm{p}^{4}\left({ }^{3} \mathrm{P}\right) 3 \mathrm{~s}{ }^{4} \mathrm{P}_{3 / 2} \rightarrow 2 \mathrm{~s}^{2} 2 \mathrm{p}^{5}{ }^{2} \mathrm{P}_{3 / 2}$ & 6.90 & $288 \pm 26$ & $271 \pm 25$ & A \\
\hline 43 & 15.89 & 15.87 & 15.87 & $\mathrm{Fe}$ & XVIII & $2 \mathrm{p}^{4}\left({ }^{1} \mathrm{D}\right) 3 \mathrm{~s}^{2} \mathrm{D}_{3 / 2} \rightarrow 2 \mathrm{~s}^{2} 2 \mathrm{p}^{5}{ }^{2} \mathrm{P}_{1 / 2}$ & 6.80 & $336 \pm 27$ & $198 \pm 24$ & \\
\hline $44 \mathrm{a}$ & 16.02 & 16.00 & 16.00 & $\mathrm{Fe}$ & XVIII & $2 \mathrm{p}^{4}\left({ }^{3} \mathrm{P}\right) 3 \mathrm{~s}^{2} \mathrm{P}_{3 / 2} \rightarrow 2 \mathrm{~s}^{2} 2 \mathrm{p}^{5}{ }^{2} \mathrm{P}_{3 / 2}$ & 6.90 & $1092 \pm 41$ & $737 \pm 35$ & \\
\hline $44 \mathrm{~b}$ & $\cdots$ & $\cdots$ & 16.01 & $\mathrm{O}$ & VIII & $3 \mathrm{p}^{2} \mathrm{P}_{3 / 2} \rightarrow 1 \mathrm{~s}{ }^{2} \mathrm{~S}_{1 / 2}$ & 6.50 & .. & .. & \\
\hline $44 c$ & $\cdots$ & $\cdots$ & 16.01 & $\mathrm{O}$ & VIII & $3 \mathrm{p}^{2} \mathrm{P}_{1 / 2} \rightarrow 1 \mathrm{~s}^{2} \mathrm{~S}_{1 / 2}$ & 6.50 & $\cdots$ & $\cdots$ & \\
\hline 45 & 16.10 & 16.07 & 16.07 & $\mathrm{Fe}$ & XVIII & $2 \mathrm{p}^{4}\left({ }^{3} \mathrm{P}\right) 3 \mathrm{~s}{ }^{4} \mathrm{P}_{5 / 2} \rightarrow 2 \mathrm{~s}^{2} 2 \mathrm{p}^{5}{ }^{2} \mathrm{P}_{3 / 2}$ & 6.90 & $1060 \pm 41$ & $820 \pm 37$ & $\mathrm{AB}$ \\
\hline 46 & 16.18 & 16.16 & 16.17 & $\mathrm{Fe}$ & XVIII & $2 \mathrm{~s} 2 \mathrm{p}^{5}\left({ }^{3} \mathrm{P}\right) 3 \mathrm{~s}^{2} \mathrm{P}_{3 / 2} \rightarrow 2 \mathrm{~s} 2 \mathrm{p}^{6}{ }^{2} \mathrm{~S}_{1 / 2}$ & 6.90 & $196 \pm 23$ & $151 \pm 20$ & \\
\hline 47 & 16.27 & 16.25 & 16.31 & $\mathrm{Fe}$ & XVIII & $2 \mathrm{~s} 2 \mathrm{p}^{5}\left({ }^{3} \mathrm{P}\right) 3 \mathrm{~s}{ }^{4} \mathrm{P}_{3 / 2} \rightarrow 2 \mathrm{~s} 2 \mathrm{p}^{6}{ }^{2} \mathrm{~S}_{1 / 2}$ & 6.90 & $141 \pm 21$ & $78 \pm 17$ & $\mathrm{AB}$ \\
\hline 48 & 16.33 & 16.31 & 16.34 & $\mathrm{Fe}$ & XVII & $2 p^{5} 3 p^{3} D_{2} \rightarrow 2 p^{6}{ }^{1} S_{0}$ & 6.70 & $156 \pm 21$ & $109 \pm 18$ & \\
\hline 49 & 16.79 & 16.76 & 16.78 & $\mathrm{Fe}$ & XVII & $2 \mathrm{p}^{5} 3 \mathrm{~s}^{3} \mathrm{P}_{1} \rightarrow 2 \mathrm{p}^{6}{ }^{1} \mathrm{~S}_{0}$ & 6.70 & $2190 \pm 52$ & $1654 \pm 45$ & $\mathrm{AB}$ \\
\hline 50 & 17.06 & 17.03 & 17.05 & $\mathrm{Fe}$ & XVII & $2 \mathrm{p}^{5} 3 \mathrm{~s}{ }^{1} \mathrm{P}_{1} \rightarrow 2 \mathrm{p}^{6}{ }^{1} \mathrm{~S}_{0}$ & 6.70 & $2471 \pm 62$ & $1856 \pm 56$ & $\mathrm{AB}$ \\
\hline 51 & 17.10 & 17.08 & 17.10 & $\mathrm{Fe}$ & XVII & $2 \mathrm{p}^{5} 3 \mathrm{~s}^{3} \mathrm{P}_{2} \rightarrow 2 \mathrm{p}^{6}{ }^{1} \mathrm{~S}_{0}$ & 6.70 & $2357 \pm 62$ & $1674 \pm 54$ & $\mathrm{AB}$ \\
\hline 52 & 17.64 & 17.61 & 17.64 & $\mathrm{Fe}$ & XVIII & $2 \mathrm{p}^{4}\left({ }^{1} \mathrm{D}\right) 3 \mathrm{p}^{2} \mathrm{P}_{3 / 2} \rightarrow 2 \mathrm{~s} 2 \mathrm{p}^{6}{ }^{2} \mathrm{~S}_{1 / 2}$ & 6.90 & $303 \pm 23$ & $252 \pm 21$ & \\
\hline 53 & 18.63 & 18.61 & 18.63 & $\mathrm{O}$ & VII & $1 \mathrm{~s} 3 \mathrm{p}^{1} \mathrm{P}_{1} \rightarrow 1 \mathrm{~s}^{2}{ }^{1} \mathrm{~S}_{0}$ & 6.35 & $140 \pm 18$ & $118 \pm 16$ & $\mathrm{AB}$ \\
\hline $54 \mathrm{a}$ & 18.97 & 18.95 & 18.97 & $\mathrm{O}$ & VIII & $2 \mathrm{p}^{2} \mathrm{P}_{3 / 2} \rightarrow 1 \mathrm{~s}^{2} \mathrm{~S}_{1 / 2}$ & 6.50 & $2309 \pm 53$ & $1827 \pm 47$ & $\mathrm{AB}$ \\
\hline $54 \mathrm{~b}$ & $\ldots$ & $\cdots$ & 18.97 & $\mathrm{O}$ & VIII & $2 \mathrm{p}^{2} \mathrm{P}_{1 / 2} \rightarrow 1 \mathrm{~s}^{2} \mathrm{~S}_{1 / 2}$ & 6.50 & 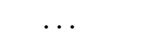 & $\ldots$ & \\
\hline 55 & 21.61 & 21.61 & 21.60 & $\mathrm{O}$ & VII & $1 \mathrm{~s} 2 \mathrm{p}{ }^{1} \mathrm{P}_{1} \rightarrow 1 \mathrm{~s}^{2}{ }^{1} \mathrm{~S}_{0}$ & 6.30 & $491 \pm 27$ & $413 \pm 25$ & $\mathrm{AB}$ \\
\hline 56 & 21.82 & 21.81 & 21.81 & $\mathrm{O}$ & VII & $1 \mathrm{~s} 2 \mathrm{p}^{3} \mathrm{P}_{1} \rightarrow 1 \mathrm{~s}^{2}{ }^{1} \mathrm{~S}_{0}$ & 6.30 & $102 \pm 15$ & $88 \pm 14$ & \\
\hline 57 & 22.11 & 22.11 & 22.10 & $\mathrm{O}$ & VII & $1 \mathrm{~s} 2 \mathrm{~s}^{3} \mathrm{~S}_{1} \rightarrow 1 \mathrm{~s}^{2}{ }^{1} \mathrm{~S}_{0}$ & 6.30 & $356 \pm 23$ & $313 \pm 22$ & \\
\hline $58 \mathrm{a}$ & 24.80 & 24.80 & 24.78 & $\mathrm{~N}$ & VII & $2 \mathrm{p}^{2} \mathrm{P}_{3 / 2} \rightarrow 1 \mathrm{~s}^{2} \mathrm{~S}_{1 / 2}$ & 6.30 & $330 \pm 23$ & $234 \pm 20$ & $\mathrm{AB}$ \\
\hline $58 \mathrm{~b}$ & $\cdots$ & $\cdots$ & 24.78 & $\mathrm{~N}$ & VII & $2 \mathrm{p}{ }^{2} \mathrm{P}_{1 / 2} \rightarrow 1 \mathrm{~s}^{2} \mathrm{~S}_{1 / 2}$ & 6.30 & $\cdots$ & $\cdots$ & \\
\hline $59 \mathrm{a}$ & 27.05 & 27.01 & 26.99 & $\mathrm{C}$ & VI & $4 \mathrm{p}^{2} \mathrm{P}_{3 / 2} \rightarrow 1 \mathrm{~s}^{2} \mathrm{~S}_{1 / 2}$ & 6.20 & $77 \pm 14$ & $54 \pm 12$ & \\
\hline $59 \mathrm{~b}$ & $\cdots$ & $\cdots$ & 26.99 & $\mathrm{C}$ & VI & $4 \mathrm{p}^{2} \mathrm{P}_{1 / 2} \rightarrow 1 \mathrm{~s}^{2} \mathrm{~S}_{1 / 2}$ & 6.20 & $\cdots$ & $\cdots$ & \\
\hline $60 \mathrm{a}$ & 28.46 & 28.42 & 28.47 & $\mathrm{C}$ & VI & $3 \mathrm{p}^{2} \mathrm{P}_{3 / 2} \rightarrow 1 \mathrm{~s}^{2} \mathrm{~S}_{1 / 2}$ & 6.20 & $119 \pm 16$ & $74 \pm 13$ & \\
\hline $60 \mathrm{~b}$ & $\cdots$ & $\cdots$ & 28.47 & $\mathrm{C}$ & VI & $3 \mathrm{p}^{2} \mathrm{P}_{1 / 2} \rightarrow 1 \mathrm{~s}^{2} \mathrm{~S}_{1 / 2}$ & 6.20 & $\cdots$ & $\cdots$ & \\
\hline 61 & 28.80 & 28.78 & 28.79 & $\mathrm{~N}$ & VI & $1 \mathrm{~s} 2 \mathrm{p}^{1} \mathrm{P}_{1} \rightarrow 1 \mathrm{~s}^{2}{ }^{1} \mathrm{~S}_{0}$ & 6.20 & $105 \pm 15$ & $91 \pm 15$ & $\mathrm{AB}$ \\
\hline 62 & 29.54 & 29.53 & 29.53 & $\mathrm{~N}$ & VI & $1 \mathrm{~s} 2 \mathrm{~s}^{3} \mathrm{~S}_{1} \rightarrow 1 \mathrm{~s}^{2}{ }^{1} \mathrm{~S}_{0}$ & 6.15 & $91 \pm 15$ & $59 \pm 13$ & \\
\hline $63 a$ & 33.74 & 33.74 & 33.73 & $\mathrm{C}$ & VI & $2 \mathrm{p}^{2} \mathrm{P}_{3 / 2} \rightarrow 1 \mathrm{~s}{ }^{2} \mathrm{~S}_{1 / 2}$ & 6.20 & $403 \pm 25$ & $286 \pm 22$ & $\mathrm{AB}$ \\
\hline $63 b$ & $\cdots$ & $\cdots$ & 33.74 & $\mathrm{C}$ & VI & $2 \mathrm{p}^{2} \mathrm{P}_{1 / 2} \rightarrow 1 \mathrm{~s}^{2} \mathrm{~S}_{1 / 2}$ & 6.20 & $\cdots$ & $\cdots$ & \\
\hline 64 & 44.03 & 44.03 & 44.02 & $\mathrm{Si}$ & XII & $1 \mathrm{~s}^{2} 3 \mathrm{~d}^{2} \mathrm{D}_{3 / 2} \rightarrow 1 \mathrm{~s}^{2} 2 \mathrm{p}^{2} \mathrm{P}_{1 / 2}$ & 6.30 & $207 \pm 20$ & $174 \pm 19$ & $\mathrm{AB}$ \\
\hline 65 & 44.18 & 44.18 & 44.17 & $\mathrm{Si}$ & XII & $1 \mathrm{~s}^{2} 3 \mathrm{~d}^{2} \mathrm{D}_{5 / 2} \rightarrow 1 \mathrm{~s}^{2} 2 \mathrm{p}^{2} \mathrm{P}_{3 / 2}$ & 6.30 & $341 \pm 23$ & $276 \pm 22$ & $\mathrm{AB}$ \\
\hline 66 & 45.51 & 45.52 & 45.52 & $\mathrm{Si}$ & XII & $1 \mathrm{~s}^{2} 3 \mathrm{~s}^{2} \mathrm{~S}_{1 / 2} \rightarrow 1 \mathrm{~s}^{2} 2 \mathrm{p}^{2} \mathrm{P}_{1 / 2}$ & 6.30 & $111 \pm 16$ & $63 \pm 14$ & $\mathrm{AB}$ \\
\hline 67 & 45.69 & 45.68 & 45.69 & $\mathrm{Si}$ & XII & $1 \mathrm{~s}^{2} 3 \mathrm{~s}^{2} \mathrm{~S}_{1 / 2} \rightarrow 1 \mathrm{~s}^{2} 2 \mathrm{p}^{2} \mathrm{P}_{3 / 2}$ & 6.30 & $169 \pm 18$ & $149 \pm 18$ & $\mathrm{AB}$ \\
\hline 68 & 49.21 & 49.21 & 49.22 & $\mathrm{Si}$ & $\mathrm{XI}$ & $2 \mathrm{~s} 3 \mathrm{~d}^{1} \mathrm{D}_{2} \rightarrow 2 \mathrm{~s} 2 \mathrm{p}{ }^{1} \mathrm{P}_{1}$ & 6.20 & $115 \pm 16$ & $74 \pm 14$ & $\mathrm{AB}$ \\
\hline $69 \mathrm{a}$ & 50.56 & 50.55 & 50.52 & $\mathrm{Si}$ & & $2 \mathrm{~s}^{2} 3 \mathrm{~d}^{2} \mathrm{D}_{3 / 2} \rightarrow 2 \mathrm{~s}^{2} 2 \mathrm{p}^{2} \mathrm{P}_{1 / 2}$ & 6.20 & $152 \pm 18$ & $122 \pm 16$ & $\mathrm{AB}$ \\
\hline $69 b$ & $\cdots$ & $\cdots$ & 50.57 & $\mathrm{Fe}$ & $\mathrm{XVI}$ & $4 \mathrm{p}^{2} \mathrm{P}_{1 / 2} \rightarrow 3 \mathrm{~s}^{2} \mathrm{~S}_{1 / 2}$ & 6.50 & $\cdots$ & $\cdots$ & \\
\hline 70 & 50.68 & 50.66 & 50.69 & $\mathrm{Si}$ & & $2 \mathrm{~s}^{2} 3 \mathrm{~d}^{2} \mathrm{D}_{5 / 2} \rightarrow 2 \mathrm{~s}^{2} 2 \mathrm{p}^{2} \mathrm{P}_{3 / 2}$ & 6.20 & $75 \pm 14$ & $57 \pm 13$ & $\mathrm{AB}$ \\
\hline 71 & 54.14 & 54.13 & 54.13 & $\mathrm{Fe}$ & $\mathrm{XVI}$ & $4 d^{2} \mathrm{D}_{3 / 2} \rightarrow 3 \mathrm{p}^{2} \mathrm{P}_{1 / 2}$ & 6.50 & $112 \pm 14$ & $66 \pm 11$ & $\mathrm{AB}$ \\
\hline 72 & 54.73 & 54.72 & 54.71 & $\mathrm{Fe}$ & $\mathrm{XVI}$ & $4 d^{2} \mathrm{D}_{5 / 2} \rightarrow 3 \mathrm{p}^{2} \mathrm{P}_{3 / 2}$ & 6.50 & $154 \pm 15$ & $103 \pm 14$ & $\mathrm{AB}$ \\
\hline
\end{tabular}


Table 1. continued.

\begin{tabular}{|c|c|c|c|c|c|c|c|c|c|}
\hline \multirow[b]{2}{*}{ Label } & \multirow[b]{2}{*}{$\lambda_{\mathrm{obs}+}^{a}$} & \multirow[b]{2}{*}{$\lambda_{\mathrm{obs}-}^{a}$} & \multicolumn{3}{|r|}{ Identification } & \multirow[b]{2}{*}{$\log T_{\max }^{b}$} & \multirow{2}{*}{$\frac{2000}{(F \pm \sigma)^{c}}$} & \multirow{2}{*}{$\frac{2001}{(F \pm \sigma)^{c}}$} & \multirow[b]{2}{*}{$e m(T)^{d}$} \\
\hline & & & $\lambda_{\text {pred }}^{a}$ & Ion & Transition ( upper $\rightarrow$ lower $)$ & & & & \\
\hline 73 & 57.88 & 57.89 & 57.88 & $\operatorname{Mg~X}$ & $1 s^{2} 3 p^{2} P_{3 / 2} \rightarrow 1 s^{2} 2 s^{2} S_{1 / 2}$ & 6.10 & $99 \pm 15$ & $52 \pm 13$ & $\mathrm{AB}$ \\
\hline 74 & 57.93 & 57.93 & 57.92 & $\operatorname{Mg~X}$ & $1 s^{2} 3 p^{2} \mathrm{P}_{1 / 2} \rightarrow 1 s^{2} 2 s^{2} S_{1 / 2}$ & 6.10 & $38 \pm 13$ & $46 \pm 13$ & $\mathrm{AB}$ \\
\hline 75 & 63.73 & 63.73 & 63.71 & Fe XVI & $4 \mathrm{~s}^{2} \mathrm{~S}_{1 / 2} \rightarrow 3 \mathrm{p}^{2} \mathrm{P}_{3 / 2}$ & 6.45 & $121 \pm 14$ & $98 \pm 13$ & \\
\hline 76 & 66.27 & 66.26 & 66.25 & Fe XVI & $4 \mathrm{f}^{2} \mathrm{~F}_{5 / 2} \rightarrow 3 \mathrm{~d}^{2} \mathrm{D}_{3 / 2}$ & 6.50 & $86 \pm 13$ & $69 \pm 12$ & $\mathrm{AB}$ \\
\hline 77 & 66.38 & 66.37 & 66.36 & Fe XVI & $4 \mathrm{f}^{2} \mathrm{~F}_{7 / 2} \rightarrow 3 \mathrm{~d}^{2} \mathrm{D}_{5 / 2}$ & 6.50 & $125 \pm 14$ & $61 \pm 12$ & $\mathrm{AB}$ \\
\hline 78 & 91.13 & 91.05 & 91.01 & Fe XIX & $2 \mathrm{~s} 2 \mathrm{p}^{5}{ }^{1} \mathrm{P}_{1} \rightarrow 2 \mathrm{~s}^{2} 2 \mathrm{p}^{4}{ }^{1} \mathrm{D}_{2}$ & 6.90 & $91 \pm 14$ & $69 \pm 13$ & \\
\hline 79 & 94.00 & 93.96 & 93.92 & Fe XVIII & $2 \mathrm{~s} 2 \mathrm{p}^{6}{ }^{2} \mathrm{~S}_{1 / 2} \rightarrow 2 \mathrm{~s}^{2} 2 \mathrm{p}^{5}{ }^{2} \mathrm{P}_{3 / 2}$ & 6.80 & $979 \pm 36$ & $725 \pm 32$ & \\
\hline 80 & 101.64 & 101.60 & 101.55 & Fe XIX & $2 \mathrm{~s} 2 \mathrm{p}^{5}{ }^{3} \mathrm{P}_{1} \rightarrow 2 \mathrm{~s}^{2} 2 \mathrm{p}^{4}{ }^{3} \mathrm{P}_{2}$ & 6.90 & $201 \pm 19$ & $139 \pm 16$ & \\
\hline 81 & 104.04 & 103.99 & 103.94 & Fe XVIII & $2 \mathrm{~s} 2 \mathrm{p}^{6}{ }^{2} \mathrm{~S}_{1 / 2} \rightarrow 2 \mathrm{~s}^{2} 2 \mathrm{p}^{5}{ }^{2} \mathrm{P}_{1 / 2}$ & 6.80 & $319 \pm 22$ & $204 \pm 19$ & \\
\hline 82 & 108.45 & 108.41 & 108.36 & Fe XIX & $2 \mathrm{~s} 2 \mathrm{p}^{5}{ }^{3} \mathrm{P}_{2} \rightarrow 2 \mathrm{~s}^{2} 2 \mathrm{p}^{4}{ }^{3} \mathrm{P}_{2}$ & 6.90 & $539 \pm 28$ & $326 \pm 22$ & \\
\hline 83 & 117.21 & 117.21 & 117.18 & Fe XXII & $1 s^{2} 2 s 2 p^{2}{ }^{2} S_{1 / 2} \rightarrow 2 s^{2} 2 p^{2} P_{1 / 2}$ & 7.10 & $107 \pm 15$ & $83 \pm 13$ & \\
\hline 84 & 118.79 & 118.77 & 118.68 & $\mathrm{Fe} x \mathrm{x}$ & $2 \mathrm{~s} 2 \mathrm{p}^{4}{ }^{4} \mathrm{P}_{1 / 2} \rightarrow 2 \mathrm{~s}^{2} 2 \mathrm{p}^{3}{ }^{4} \mathrm{~S}_{3 / 2}$ & 7.00 & $100 \pm 14$ & $90 \pm 14$ & \\
\hline 85 & 120.08 & 120.08 & 119.98 & Fe XIX & $2 \mathrm{~s} 2 \mathrm{p}^{5}{ }^{3} \mathrm{P}_{2} \rightarrow 2 \mathrm{~s}^{2} 2 \mathrm{p}^{4}{ }^{3} \mathrm{P}_{1}$ & 6.90 & $136 \pm 16$ & $97 \pm 14$ & \\
\hline 86 & 121.95 & 121.93 & 121.84 & Fe $\mathrm{XX}$ & $2 \mathrm{~s} 2 \mathrm{p}^{4}{ }^{4} \mathrm{P}_{3 / 2} \rightarrow 2 \mathrm{~s}^{2} 2 \mathrm{p}^{3}{ }^{4} \mathrm{~S}_{3 / 2}$ & 7.00 & $191 \pm 18$ & $120 \pm 15$ & \\
\hline 87 & 128.85 & 128.81 & 128.75 & Fe XXI & $2 \mathrm{~s} 2 \mathrm{p}^{3}{ }^{3} \mathrm{D}_{1} \rightarrow 2 \mathrm{p}^{2}{ }^{3} \mathrm{P}_{0}$ & 7.00 & $125 \pm 15$ & $78 \pm 13$ & \\
\hline 88 & 132.94 & 132.92 & 132.84 & $\mathrm{Fe} \mathrm{xx}$ & $2 \mathrm{~s} 2 \mathrm{p}^{4}{ }^{4} \mathrm{P}_{5 / 2} \rightarrow 2 \mathrm{~s}^{2} 2 \mathrm{p}^{3}{ }^{4} \mathrm{~S}_{3 / 2}$ & 7.00 & $141 \pm 18$ & $130 \pm 17$ & \\
\hline 89 & 132.98 & 132.97 & 132.91 & Fe XXIII & $2 \mathrm{~s} 2 \mathrm{p}{ }^{1} \mathrm{P}_{1} \rightarrow 2 \mathrm{~s}^{2}{ }^{1} \mathrm{~S}_{0}$ & 7.10 & $150 \pm 18$ & $110 \pm 16$ & \\
\hline 90 & 135.91 & 135.91 & 135.76 & Fe XXII & $1 \mathrm{~s}^{2} 2 \mathrm{~s} 2 \mathrm{p}^{2}{ }^{2} \mathrm{D}_{3 / 2} \rightarrow 2 \mathrm{~s}^{2} 2 \mathrm{p}^{2} \mathrm{P}_{1 / 2}$ & 7.10 & $55 \pm 12$ & $59 \pm 12$ & \\
\hline 91 & 171.16 & 171.16 & 171.07 & Fe IX & $3 \mathrm{p}^{5} 3 \mathrm{~d}^{1} \mathrm{P}_{1} \rightarrow 3 \mathrm{p}^{6}{ }^{1} \mathrm{~S}_{0}$ & 5.80 & $74 \pm 12$ & $58 \pm 10$ & $\mathrm{AB}$ \\
\hline
\end{tabular}

${ }^{a}$ Observed (for each order of the spectrum taken in 2000) and predicted (CHIANTI database) wavelengths in $\AA$. In the cases of unresolved blends, identified by the same label number, we list the main components in order of increasing predicted wavelength.

${ }^{b}$ Temperature of maximum emissivity in $\mathrm{K}$.

${ }^{c}$ Total line counts for each observation. In the cases of unresolved blends, identified by the same label number, we report only the total line counts.

${ }^{d}$ Lines selected to derive the emission measure distribution for the observation taken in 2000 (A) or 2001 (B).

approximated with a linear function in the proximity of each line; we have checked the agreement between the predicted continuum level and the one used for line flux evaluation after reconstruction of the emission measure distribution, $\mathrm{em}(T)$, and abundances computation. In case of line blends we have performed a multi-component fitting, and in all cases we have evaluated statistical uncertainties on $\lambda_{\mathrm{c}}$ and $F$ at the $68 \%$ confidence level.

We have found the presence of small differences in the wavelength calibration between the positive and negative order spectra, yielding relative differences in the line centroids of $\leq 0.04 \AA$, and also absolute differences with respect to the CHIANTI wavelengths up to $0.1 \AA$ but only for lines with $\lambda \geq 90 \AA$. In order to get over these calibration problems we have fitted separately the positive and negative spectra, and then we have summed the counts to obtain the total line counts. Given the high count rate of the source, this procedure causes only a small reduction of the signal to noise ratio on individual lines measurements.

\section{Results}

\subsection{Line identification}

We have identified $\mathrm{Fe}$ lines with ionization level ranging from Fe XVI up to Fe XXIV, plus the Fe IX line at $171 \AA$. We have also identified $\mathrm{C}, \mathrm{N}, \mathrm{O}, \mathrm{Ne}, \mathrm{Mg}, \mathrm{Si}$, and $\mathrm{Ni}$ lines. We have cross-checked our line list with those reported by Brinkman et al. (2000), Mewe et al. (2001), Phillips et al. (2001), Behar et al. (2001) and we have found overall consistency, with few exceptions possibly due to the different line databases adopted by us and by the other authors. In particular our identifications in the region between 10 and $12 \AA$ are sometimes at variance from what reported by Phillips et al. (2001) and Behar et al. (2001): in fact we ascribe most of the spectral features observed to highly ionized iron lines (mainly Fe XXIIXXIV), supported by the recent experimental results reported by Brown et al. (2002) and by the line identifications reported by Ayres et al. (2001), while Phillips et al. and Behar et al. ascribe the same features to Fe XVII-XIX lines. Most of these lines are 


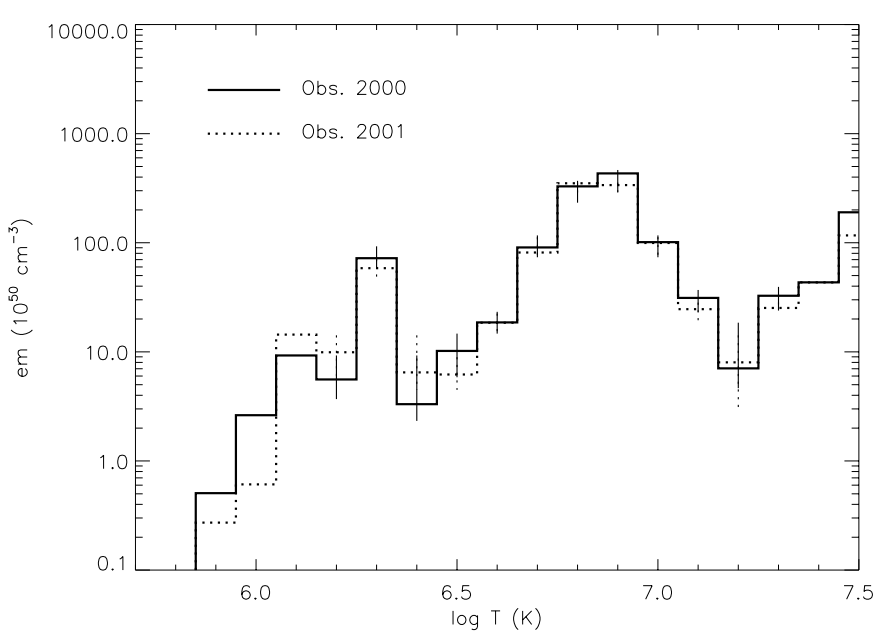

Fig. 2. Emission measure distributions calculated with the MCMC method by Kashyap \& Drake (1998) for the observation made on March 2000 (solid line) and for the observation made on February 2001 (dotted line). Note that we can provide error bars only for the bins ranging from $\log T=6.2$ to $\log T=7.3$.

not included in the CHIANTI database because they originate from highly excited levels $(n \geq 4)$. However we have verified that our results (Sect. 3.2) are not significantly affected by the lack of these lines in our analysis. More details about this issue are reported in Appendix A.

\subsection{Emission measure distribution}

Under the condition of optically-thin plasma the line photon flux relative to the transition of an ion between the levels $i \rightarrow f$, can be written as ${ }^{1}$ :

$F_{i f}=\frac{1}{4 \pi D^{2}} \int_{T} G_{i f}\left(T, N_{\mathrm{e}}\right) \operatorname{dem}(T) \mathrm{d} \log T$

where $D$ is the star distance, $G_{i f}\left(T, N_{\mathrm{e}}\right)$ is the line contribution function and $\operatorname{dem}(T)$ is the differential emission measure, defined as:

$\operatorname{dem}(T)=N_{\mathrm{e}}^{2} \frac{\mathrm{d} V(T)}{\mathrm{d} \log T}$.

We have reconstructed the emission measure distribution from measured line fluxes using the Markov-Chain Monte Carlo (hereafter MCMC) method by Kashyap \& Drake (1998), which allows us to estimate also the element abundances at the same time; this method has the additional advantage, over other procedures, of yielding uncertainties on the emission measure at various temperatures and on abundances ${ }^{2}$.

1 The exact relation between observed line counts and source emission measure contains three other factors due to instrumental effective area, exposure time and interstellar medium absorption. The first and the second are known, while for the latter we have assumed $N_{H}=1.8 \times 10^{18} \mathrm{~cm}^{-2}$ (Linsky et al. 1993).

2 As a matter of fact we can derive the product between the emission measure distribution and the iron abundance and we evaluate the abundance of the other elements as ratios with respect to the iron abundance.
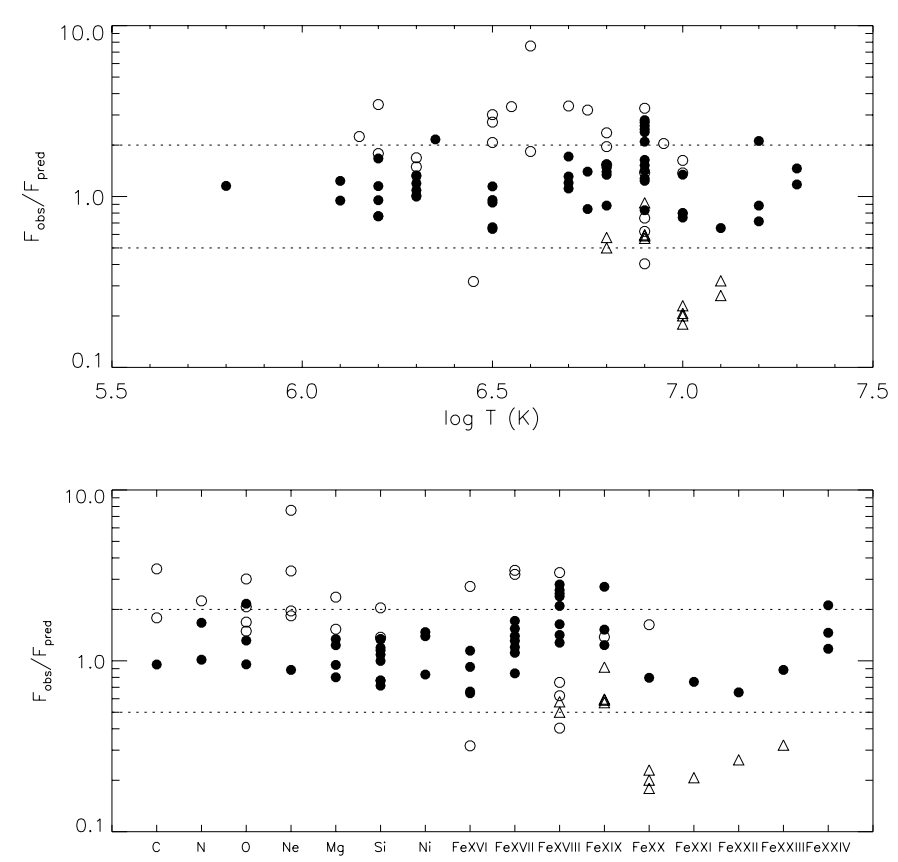

Fig. 3. Comparison between observed and predicted line fluxes vs. $T_{\max }$ (upper panel) and vs. chemical elements and, for iron, ionization stage (lower panel). Filled symbols indicate the lines included in the emission measure reconstruction, open symbols indicate those not included, and triangles indicate the long wavelength lines $(\lambda>80 \AA)$.

The differential emission measure $\operatorname{dem}(T)$ defined by Eq. (3) is a continuous function of temperature $T$; the MCMC method yields the volume emission measure distribution $\operatorname{em}\left(T_{n}\right)$ defined over a pre-selected temperature grid; in our case the grid is characterized by $\Delta \log T=0.1$ and ranges from $\log T=5.7$ to $\log T=7.5$. The final set of $T_{n}$ over which the evaluation of uncertainties is possible depends on the temperatures of peak emissivity, $T_{\max }$, of the used lines: we can compute the $\operatorname{em}\left(T_{n}\right)$ value, with its uncertainty, only if we have a sufficient number of lines with $T_{\max } \approx T_{n}$, i.e. enough information about the plasma emission at this temperature. In practice we are able to constrain the $\operatorname{em}\left(T_{n}\right)$ between $\log T=6.2$ and $\log T=7.3$.

Before starting the MCMC procedure it is necessary to select a subset of the identified lines which is used to derive the emission measure distribution. We aimed at using only those lines that are most reliable, i.e. not blended, with sufficiently high signal to noise ratio and with flux compatible with that of other lines of the same element. Moreover we have eliminated density-sensitive lines otherwise the $\operatorname{em}(T)$ derivation would be influenced by unrelated density effects; thanks to this choice the remaining lines have contribution functions which are only temperature dependent. It is worth noting that some subjectivity is introduced with the selection itself, but given the uncertainty of some of the atomic physics models and of some of the line measurements we must use only spectral information that we consider reliable.

In the last column of Table 1 we have indicated the lines selected for the $\operatorname{em}\left(T_{j}\right)$ reconstruction. Note that we have excluded all the lines with $\lambda>80 \AA$, except the Fe IX line at $171 \AA$, because, for each iron ion, the emission measure 

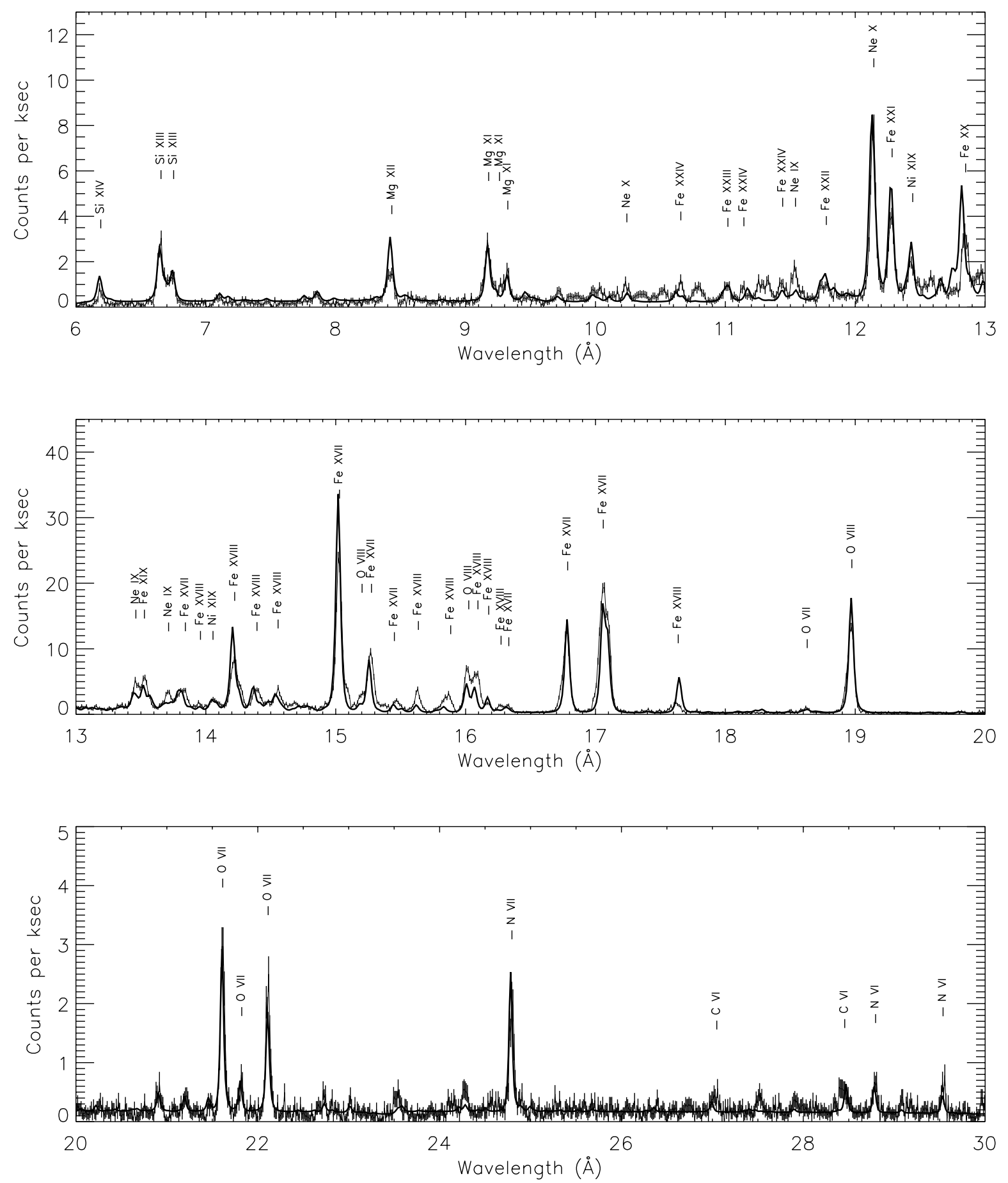

Fig. 4. Comparison between LETGS spectrum of Capella (with error bars), collected on March 8, 2000, and the predicted spectrum (thick line) derived from the reconstructed emission measure distribution.

distribution provided by short wavelength lines is significantly and consistently higher than that provided by long wavelength ones by a factor comprised between 2 and 5 . We have decided to base our emission measure analysis on short wavelength lines because they are more numerous and cover a larger temperature range than the remaining long wavelength lines that we have excluded. In Appendix B we report a commented list of the selected lines.

Finally we emphasize that our identifications of the hot iron lines with $10 \AA<\lambda<12 \AA$ (discussed in Sect. 3.1 and in Appendix A) are confirmed by the agreement between the emission measure provided by these lines and by other lines with similar $T_{\max }$.

The $\operatorname{em}(T)$ distributions, derived for each observation, are shown in Fig. 2, where we have adopted $D=12.93 \mathrm{pc}$ (Perryman et al. 1997). In order to check the quality of our result we have computed the line fluxes for all the identified lines in the spectrum and, in Fig. 3 we show the comparison between predicted and observed fluxes for the observation taken 
Table 2. Element abundances computed with the MCMC method.

\begin{tabular}{|c|c|c|c|c|c|}
\hline & \multirow[b]{2}{*}{$n^{a}$} & \multicolumn{2}{|c|}{ Obs. 2000} & \multicolumn{2}{|c|}{ Obs. 2001} \\
\hline & & $N(X) / N(H)^{b}$ & $N(X) / N(X)_{\odot}^{c}$ & $N(X) / N(H)^{b}$ & $N(X) / N(X)_{\odot}^{c}$ \\
\hline $\mathrm{C}$ & 1 & $\left(2.7_{-}^{+} \begin{array}{c}0.5 \\
0.7\end{array}\right) \times 10^{-4}$ & $0.76_{-0.18}^{+0.15}$ & $(2.5+0.7) \times 10^{-4}$ & $0.71_{-0.13}^{+0.20}$ \\
\hline $\mathrm{N}$ & 2 & $\left(1.0_{-}^{+} \begin{array}{c}0.3 \\
0.2\end{array}\right) \times 10^{-4}$ & $1.07_{-0.20}^{+0.30}$ & $\left(1.02_{-0.21}^{+0.27}\right) \times 10^{-4}$ & $1.10_{-0.22}^{+0.29}$ \\
\hline $\mathrm{O}$ & 3 & $\left(\begin{array}{ll}3.4_{-}^{+} & 0.1 \\
0.8\end{array}\right) \times 10^{-4}$ & $0.46_{-0.11}^{+0.01}$ & $(3.7+0.4) \times 10^{-4}$ & $0.50_{-0.06}^{+0.05}$ \\
\hline $\mathrm{Ne}$ & 1 & $\left(\begin{array}{cc}6.0_{-}^{+} & 0.5 \\
0.8\end{array}\right) \times 10^{-5}$ & $0.50_{-0.07}^{+0.04}$ & $(6.0+0.4) \times 10^{-5}$ & $0.50_{-0.08}^{+0.03}$ \\
\hline $\mathrm{Mg}$ & 4 & $\left(3.4_{-}^{+} \quad 0.7\right) \times 10^{-5}$ & $0.88_{-0.21}^{+0.19}$ & $\left(3.6+\begin{array}{c}0.5 \\
1.0\end{array}\right) \times 10^{-5}$ & $0.94_{-0.26}^{+0.14}$ \\
\hline $\mathrm{Si}$ & 9 & $\left(3.1_{-}^{+} \quad 1.0\right) \times 10^{-5}$ & $0.87_{-0.15}^{+0.28}$ & $\left(\begin{array}{ll}3.4+0.7 \\
-\end{array}\right) \times 10^{-5}$ & $0.95_{-0.22}^{+0.20}$ \\
\hline $\mathrm{Ni}$ & 3 & $\left(2.6_{-}^{+} 0.6\right) \times 10^{-6}$ & $1.5_{-}^{+} 0.3$ & $(2.8+0.4) \times 10^{-6}$ & $1.6_{-0.5}^{+0.22}$ \\
\hline$\overline{\mathrm{Fe}^{d}}$ & - & $3.24 \times 10^{-5}$ & 1 & $3.24 \times 10^{-5}$ & 1 \\
\hline
\end{tabular}

${ }^{a}$ Number of spectral lines of the relevant element included in the emission measure reconstruction.

${ }^{b}$ Element abundance relative to hydrogen.

${ }^{c}$ Element abundances relative to the solar ones (Grevesse et al. 1992).

${ }^{d}$ Coronal abundance equal to the solar value (Grevesse et al. 1992) as determined by adjustment of the continuum (see Sect. 3.3).

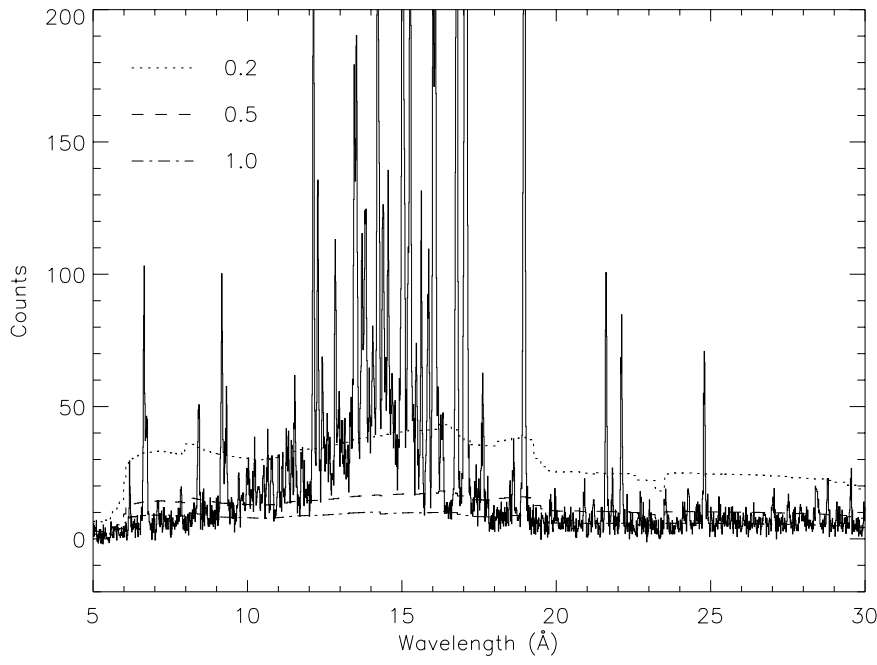

Fig. 5. Comparison between observed spectrum (solid line) and predicted continuum spectra with different iron abundances (reported in the legend) relative to the Grevesse et al. (1992) solar value. Predicted continuum spectra are generated using the $\operatorname{em}(T)$ and abundance values derived by the MCMC procedure, and $N_{\mathrm{e}}=10^{10} \mathrm{~cm}^{-3}$. The best determination of the relative line to continuum ratio is achieved assuming a coronal metal abundance equal to the solar one.

in 2000. The results obtained for the observation taken in 2001 are similar.

Figure 3 shows that for most lines there is agreement between predicted and observed fluxes within a factor 2 , at $T=10^{6.9} \mathrm{~K}$ there is a large spread due to Fe XVIII lines, while the long wavelength lines (triangles) have observed fluxes systematically lower than predicted, as already anticipated above. Finally, in Fig. 4 we compare the observed spectrum and the spectrum predicted on the basis of the $\operatorname{em}(T)$ distribution we have derived, in the spectral region between 6 and $30 \AA$. The agreement is reasonably good, except in the region between 10 and $12 \AA$ where the CHIANTI database does not contain the lines required to describe some of the observed spectral features (see Sect. 3.1 and Appendix A), and for some Fe XVIII lines affected by uncertainties in the CHIANTI database.

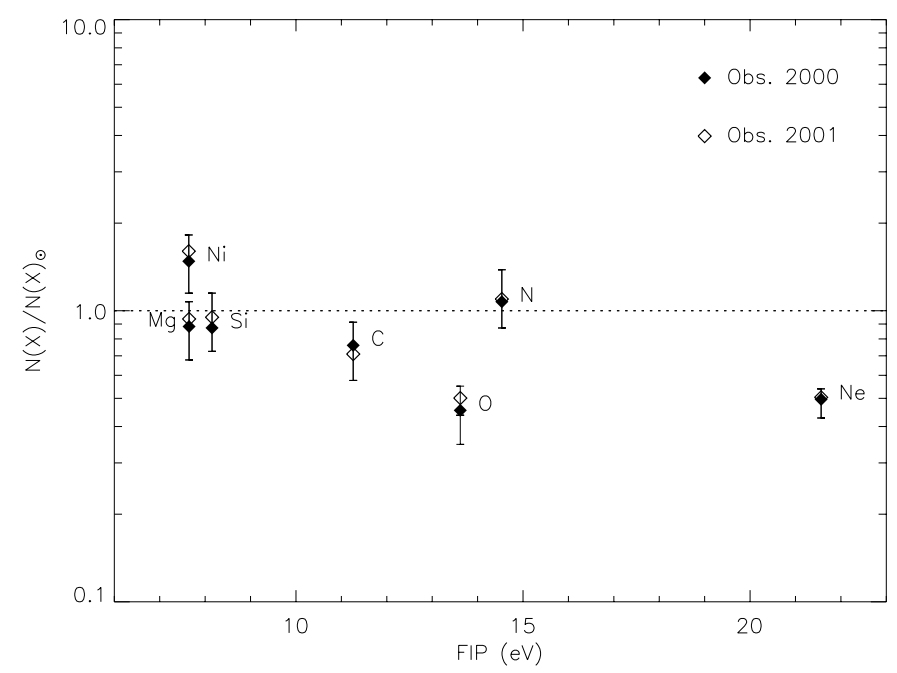

Fig. 6. Derived abundances, in solar units (Grevesse et al. 1992), vs. first ionization potential. Filled diamonds are abundances derived from the observation taken on March 2000, open diamonds the ones derived from the observation taken on February 2001.

The $e m(T)$ distributions that we have derived show a sharp peak near $\log T=6.8-6.9$, in agreement with results obtained by Dupree et al. (1993), Schrijver et al. (1995) and Brickhouse et al. (2000), who derived the emission measure distribution using EUVE data. A little amount of plasma is found at temperatures higher than $10^{7} \mathrm{~K}$ (note however that the $\operatorname{em}(T)$ at $\log T=7.4-7.5$ is not constrained by error bars). These results suggest that the hot tail of emission measure distribution of Capella is larger than what found by Dupree et al. (1999) from an EUVE observation made in 1999.

By comparing the $e m(T)$ obtained from the two spectra we can assert that the corona of Capella was approximately in the same $\operatorname{em}(T)$ conditions after a period of 11 months.

We have derived the coronal global abundance of Capella by scaling the element abundances for various metallicities, and consequently the emission measure distribution, and then by comparing the observed spectrum with the predicted 

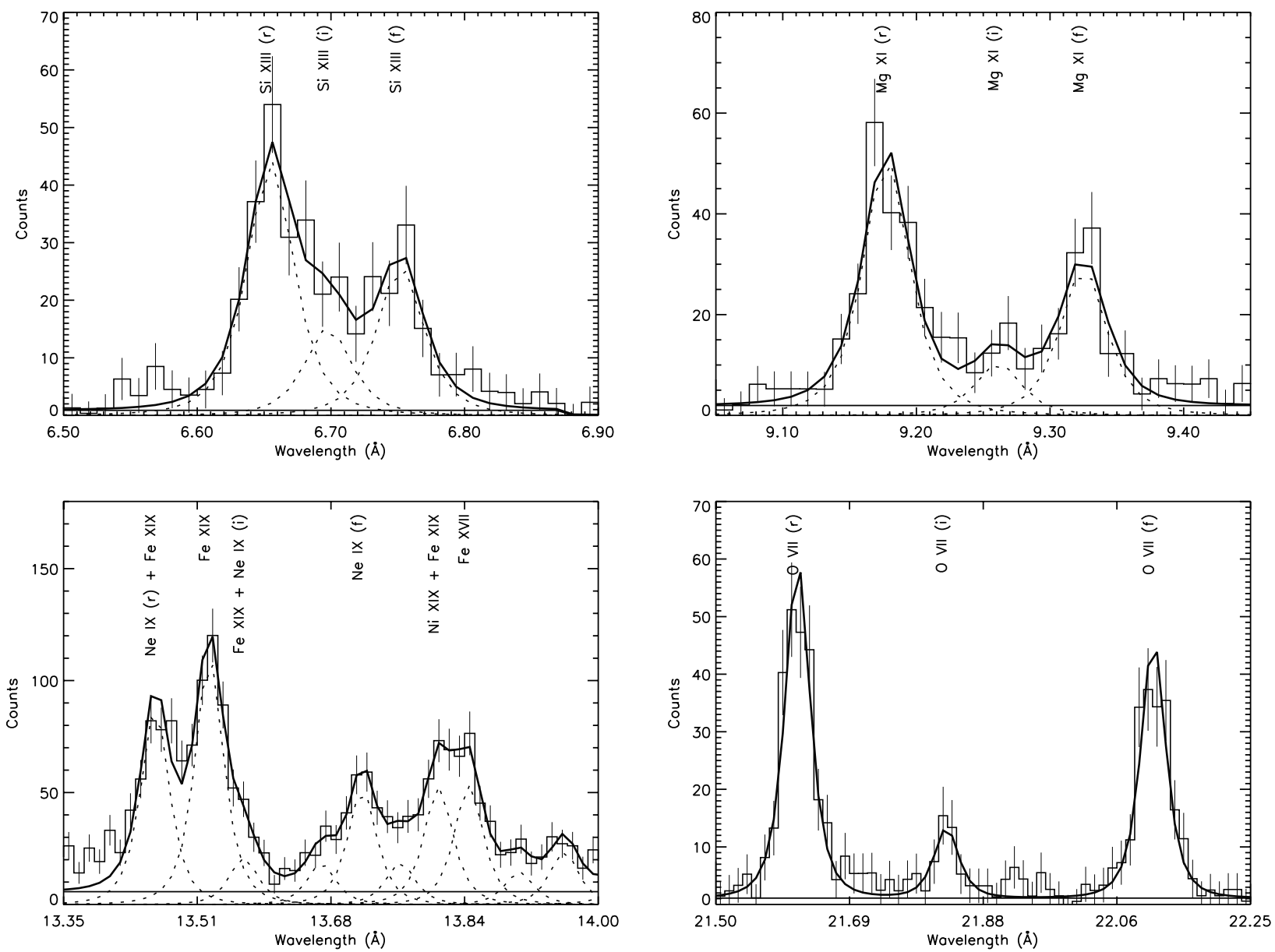

Fig. 7. Observed and best-fit multi-component model spectrum for the Si XIII, Mg XI, Ne IX and O VII triplets of the positive order of the observation taken on March 2000. Dotted lines represent individual lines while the horizontal line indicates the continuum.

continuum level. To perform this check it is important to evaluate the continuum level without any influence of unresolved weak lines, therefore we have analyzed spectral regions which are free of emission lines according to the CHIANTI database. In Fig. 5 we show the predicted continuum spectra for different iron abundances relative to solar values superimposed to the observed one.

\subsection{Metallicity and abundances}

We have found that the coronal iron abundance of Capella is very similar to the solar photospheric value ${ }^{3}$. Bauer \& Bregman (1996) have determined a global metal abundance of Capella of 0.2 times the solar one from a ROSAT observation. The disagreement observed in the global abundances between our value and the one by Bauer \& Bregman may be related to the different resolutions of the studied spectra. Favata et al. (1997), analyzing data from $S A X$, have evaluated the value $0.98 \pm 0.07$, this result agrees with the values of $1.27 \pm 0.19$ and $1.04 \pm 0.09$ derived by Brickhouse et al. (2000) based on observations of

\footnotetext{
${ }^{3}$ We use as reference value the Fe solar abundance reported by Grevesse et al. (1992), therefore we have converted metallicity reported by other authors to this one.
}

$E U V E$ and $A S C A$ respectively and with the values of $0.90 \pm 0.03$ by Audard et al. (2001) obtained by analysis of the XMM RGS1 spectrum: all these findings are compatible with our determination. On the other hand the value $0.74 \pm 0.01$ derived by Audard et al. (2001) from the XMM RGS2 spectrum is rather small.

In Table 2 we report the element abundances obtained from each spectrum and the number of lines of each element included in the reconstruction of the emission measure distribution. In Fig. 6 we have plotted the derived abundances in solar units (Grevesse et al. 1992) versus the first ionization potential (FIP). We use the solar photospheric abundances as reference values because the Capella photospheric abundances are not known individually. The $\mathrm{C}, \mathrm{N}, \mathrm{Mg}, \mathrm{Si}$ and $\mathrm{Ni}$ abundances are consistent (C and $\mathrm{Ni}$ within two standard deviations) with solar values, while $\mathrm{O}$ and $\mathrm{Ne}$ abundances are a factor 0.5 the solar ones.

Brickhouse et al. (2000) have found abundances of $\mathrm{Ne}, \mathrm{Mg}$, $\mathrm{Si}$ (from $A S C A$ data), $\mathrm{O}$ and $\mathrm{Ni}$ (from $E U V E$ data), compatible with our values, while the Si abundance derived from $E U V E$ results larger by a factor 2 . The abundances reported by Audard et al. (2001) agree with our results except for the case of Si and $\mathrm{Ni}$ for which the Audard et al. values are lower by a factor 2 . 
Table 3. Density and temperature determination, from the line ratios $R_{\mathrm{D}}=f / i$ and $R_{\mathrm{T}}=(i+f) / r$, for the analysed He-like triplets.

\begin{tabular}{|c|c|c|c|c|c|}
\hline \multicolumn{6}{|c|}{ Obs. 2000} \\
\hline Ion & $R_{\mathrm{D}}$ & $N_{\mathrm{e}}$ & $R_{\mathrm{T}}$ & $\log T(\mathrm{~K})$ & $\log T_{\max }(\mathrm{K})$ \\
\hline O VII & $3.5 \pm 0.6$ & $<1 \times 10^{10} \mathrm{~cm}^{-3}$ & $0.95 \pm 0.08$ & $6.22 \pm 0.08$ & 6.3 \\
\hline Ne IX & $3.2 \pm 0.8$ & $<2 \times 10^{11} \mathrm{~cm}^{-3}$ & $1.08 \pm 0.10$ & $6.21 \pm 0.12$ & 6.6 \\
\hline Mg XI & $2.2 \pm 0.4$ & $\left(2.0_{-0.9}^{+2.0}\right) \times 10^{12} \mathrm{~cm}^{-3}$ & $0.79 \pm 0.08$ & $6.64 \pm 0.09$ & 6.8 \\
\hline Si XIII & $2.0 \pm 0.5$ & $<2.0 \times 10^{13} \mathrm{~cm}^{-3}$ & $0.73 \pm 0.08$ & $6.78 \pm 0.11$ & 7.0 \\
\hline \multicolumn{6}{|c|}{ Obs. 2001} \\
\hline Ion & $R_{\mathrm{D}}$ & $N_{\mathrm{e}}$ & $R_{\mathrm{T}}$ & $\log T(\mathrm{~K})$ & $\log T_{\max }(\mathrm{K})$ \\
\hline O VII & $3.6 \pm 0.6$ & $<1 \times 10^{10} \mathrm{~cm}^{-3}$ & $0.98 \pm 0.09$ & $6.18 \pm 0.08$ & 6.3 \\
\hline Ne IX & $2.5 \pm 0.6$ & $\left(1.8_{-1.3}^{+2.5}\right) \times 10^{11} \mathrm{~cm}^{-3}$ & $1.00 \pm 0.10$ & $6.29 \pm 0.12$ & 6.6 \\
\hline Mg XI & $2.4 \pm 0.5$ & $\left(1.5_{-13}^{+1.7}\right) \times 10^{12} \mathrm{~cm}^{-3}$ & $0.87 \pm 0.09$ & $6.54 \pm 0.12$ & 6.8 \\
\hline Si XIII & $3.1 \pm 1.0$ & $<9 \times 10^{12} \mathrm{~cm}^{-3}$ & $0.78 \pm 0.11$ & $6.71 \pm 0.14$ & 7.0 \\
\hline
\end{tabular}

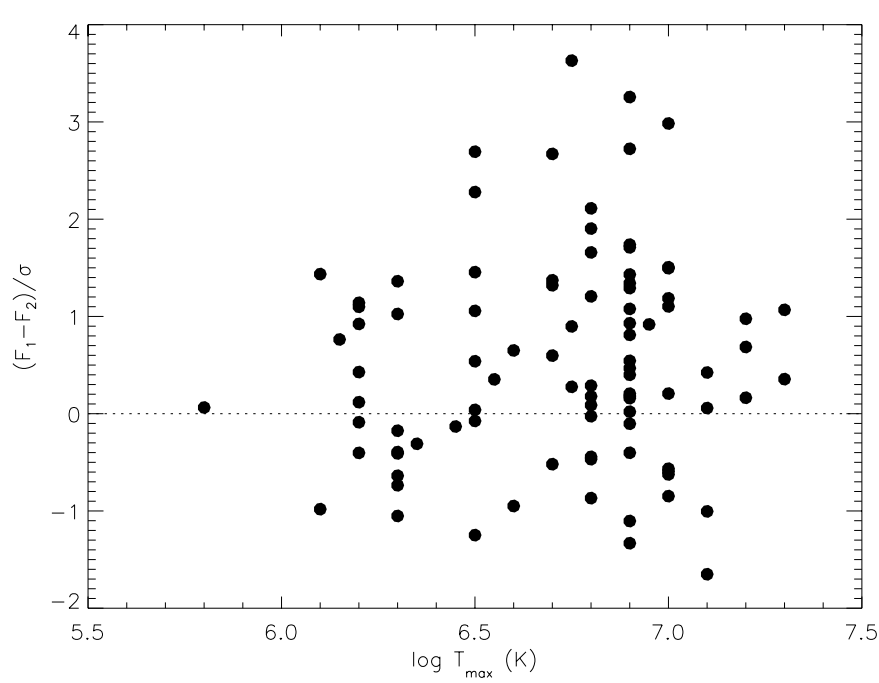

Fig. 8. Comparison, in $\sigma$ unit, between observed line fluxes of the two observations.

\subsection{Density and temperature diagnostics from He-like ions}

The relative intensity of the resonance, intercombination and forbidden lines (hereafter $r, i, f$ ) of the helium-like ions allows us to derive the plasma temperature, $T$, and electron density, $N_{\mathrm{e}}$, averaged over the region in which the lines are formed (Gabriel \& Jordan 1969; Porquet et al. 2001). In particular the density is determined by the ratio $R_{\mathrm{D}}=f / i$, while the temperature by the ratio $R_{\mathrm{T}}=(i+f) / r$.

The wavelength range of the LETGS includes the heliumlike triplets of the ions $\mathrm{CV}, \mathrm{N}$ VI, O VII, Ne IX, Mg XI and $\mathrm{Si}$ XIII, thus allowing to sample densities over the temperature range $10^{6}-10^{7} \mathrm{~K}$. We have analyzed only the last four triplets, because the others are either too weak or affected by contamination by high-order spectra (see Ness et al. 2001, for the analysis of the $\mathrm{C} \mathrm{V}$ and $\mathrm{N}$ VI triplets). The Mg XI and Si XIII triplets are partially blended, but it is possible to evaluate the fluxes of individual lines with a multi-component fitting. In Fig. 7 the observed and best-fit model spectra are shown.

The analysis of the Ne IX lines is especially difficult because of severe blending with Fe XIX lines (see the

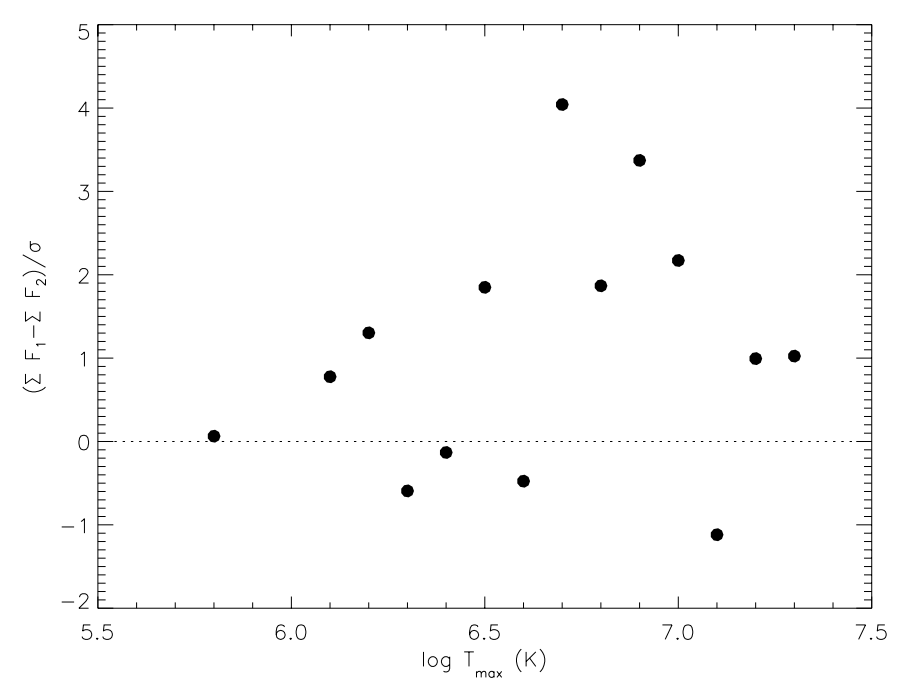

Fig. 9. Comparison, in $\sigma$ unit, between total observed line fluxes at each $T_{\max }$ of the two observations.

identifications from 20 to 29 in Table 1). It is worth noting that with the fitting procedure we are able to determine the total flux of each spectral feature, but not the flux of the unresolved line components which contribute to it. Therefore in order to perform the density and temperature diagnostics with the Ne IX triplet we need to derive the flux of the $r$ and $i$ Ne IX lines from the measured spectral features which include them. In the case of the resonance Ne IX line it is straightforward to use the emission measure distribution and the $\mathrm{Ne} / \mathrm{Fe}$ abundance ratio to evaluate the relative intensities of the Ne IX and Fe XIX line components, and hence to derive the intensity of the $r$ line of Ne IX from the measured total flux. Instead, in order to obtain the flux of the density-dependent intercombination line we have simply subtracted the predicted flux of the Fe XIX $13.57 \AA$ line component from the measured total flux of the spectral feature \# 22 of Table 1.

In Table 3 we report the $R_{\mathrm{D}}$ and $R_{\mathrm{T}}$ values for each ion and each spectrum, and the derived values of $N_{\mathrm{e}}$ and $T$. For all the triplets we have found a good consistency (within $1 \sigma$ uncertainties) of the results obtained from the two spectra. Moreover, the values of the $R_{\mathrm{D}}$ and $R_{\mathrm{T}}$ ratios are compatible 
Table 4. Results for the short-term variability analysis. We report only upper limits for the effective variability at the $99.7 \%$ statistical level, because the emission is compatible with the hypothesis of constant source in the cases.

\begin{tabular}{|c|c|c|c|c|c|c|}
\hline \multirow[b]{2}{*}{$\log T_{\max }^{a}$} & \multirow[b]{2}{*}{$\Delta \lambda^{b}$} & \multirow[b]{2}{*}{ Ion } & \multicolumn{2}{|c|}{ Obs. 2000} & \multicolumn{2}{|c|}{ Obs. 2001} \\
\hline & & & $N_{\text {TOT }}^{c}$ & $V_{\text {eff }} / I^{d}(\%)$ & $N_{\mathrm{TOT}}^{c}$ & $V_{\text {eff }} / I^{d}(\%)$ \\
\hline \multirow[t]{4}{*}{6.30} & {$[21.50,22.25]$} & O VII & 2557 & $<10.3$ & 2128 & $<10.8$ \\
\hline & {$[24.75,24.85]$} & N VII & & & & \\
\hline & {$[43.98,44.25]$} & Si XII & & & & \\
\hline & {$[45.58,45.75]$} & Si XII & & & & \\
\hline \multirow[t]{2}{*}{6.50} & {$[15.95,16.05]$} & O VIII & 3710 & $<8.6$ & 2797 & $<9.4$ \\
\hline & {$[18.85,19.05]$} & O VIII & & & & \\
\hline \multirow[t]{4}{*}{6.70} & {$[14.95,15.12]$} & Fe XVII & 13258 & $<4.5$ & 9966 & $<5.0$ \\
\hline & {$[15.22,15.33]$} & Fe XVII & & & & \\
\hline & {$[16.70,16.90]$} & Fe XVII & & & & \\
\hline & {$[16.95,17.20]$} & Fe XVII & & & & \\
\hline \multirow[t]{4}{*}{6.80} & {$[9.05,9.45]$} & Mg XI & 3891 & $<8.4$ & 2980 & $<9.1$ \\
\hline & {$[12.05,12.22]$} & $\mathrm{NeX}$ & & & & \\
\hline & {$[93.90,94.10]$} & Fe XVIII & & & & \\
\hline & {$[103.93,104.12]$} & Fe XVIII & & & & \\
\hline \multirow[t]{7}{*}{6.90} & {$[14.15,14.65]$} & Fe XVIII & 8005 & $<5.8$ & 5841 & $<6.5$ \\
\hline & {$[15.55,15.70]$} & Fe XVIII & & & & \\
\hline & {$[15.80,15.93]$} & Fe XVIII & & & & \\
\hline & {$[16.05,16.20]$} & Fe XVIII & & & & \\
\hline & {$[17.58,17.66]$} & Fe XVIII & & & & \\
\hline & {$[101.55,101.70]$} & Fe XIX & & & & \\
\hline & {$[108.37,108.48]$} & Fe XIX & & & & \\
\hline \multirow[t]{8}{*}{7.00} & {$[6.55,6.85]$} & Si XIII & 3493 & $<8.9$ & 2465 & $<10.0$ \\
\hline & {$[8.30,8.50]$} & Mg XII & & & & \\
\hline & {$[12.22,12.35]$} & Fe XXI & & & & \\
\hline & {$[12.80,12.90]$} & Fe $X X$ & & & & \\
\hline & {$[118.70,118.85]$} & Fe XX & & & & \\
\hline & {$[121.85,122.00]$} & $\mathrm{Fe} X \mathrm{X}$ & & & & \\
\hline & {$[128.75,128.92]$} & Fe XXI & & & & \\
\hline & {$[132.85,133.05]$} & Fe XXIII & & & & \\
\hline
\end{tabular}

\footnotetext{
${ }^{a}$ Peak temperature of line formation.
}

${ }^{b}$ Wavelength interval used to select photons. For each $T_{\max }$ value, we have analyzed together the photons extracted from all the listed wavelength intervals.

${ }^{c}$ Total number of selected photons, including the background.

${ }^{d}$ Effective fractional variability.

with those determined from the analysis of other Capella spectra obtained with Chandra or XMM-Newton (Brinkman et al. 2000; Canizares et al. 2000; Ness et al. 2001; Mewe et al. 2001; Phillips et al. 2001; Audard et al. 2001; Ness et al. 2002). Note however that, in some cases, the use of different models (Pradhan 1982, SPEX/MEKAL, Porquet \& Dubau 2000 and Keenan et al. 1984) produces small differences for $T$ (within a factor 2) and $N_{\mathrm{e}}$ (within a factor 5).

It is worth noting that all the temperatures we have found are lower than the corresponding temperatures of maximum line emissivity $\left(T_{\max }\right)$. This behavior will be commented in Sect. 4.

\subsection{Variability}

We have tested the presence of variability in the X-ray emission from Capella over different time scales and using different approaches: in order to look for variability on long-time scales we have compared the total count rates measured in the two observations, and we have compared the measured line counts of individual lines or groups of lines which form at similar $T$; moreover we have performed a detailed variability analysis on the light curves of individual emission lines, within each observation, with the aim to test for the presence of any short-term variability. 

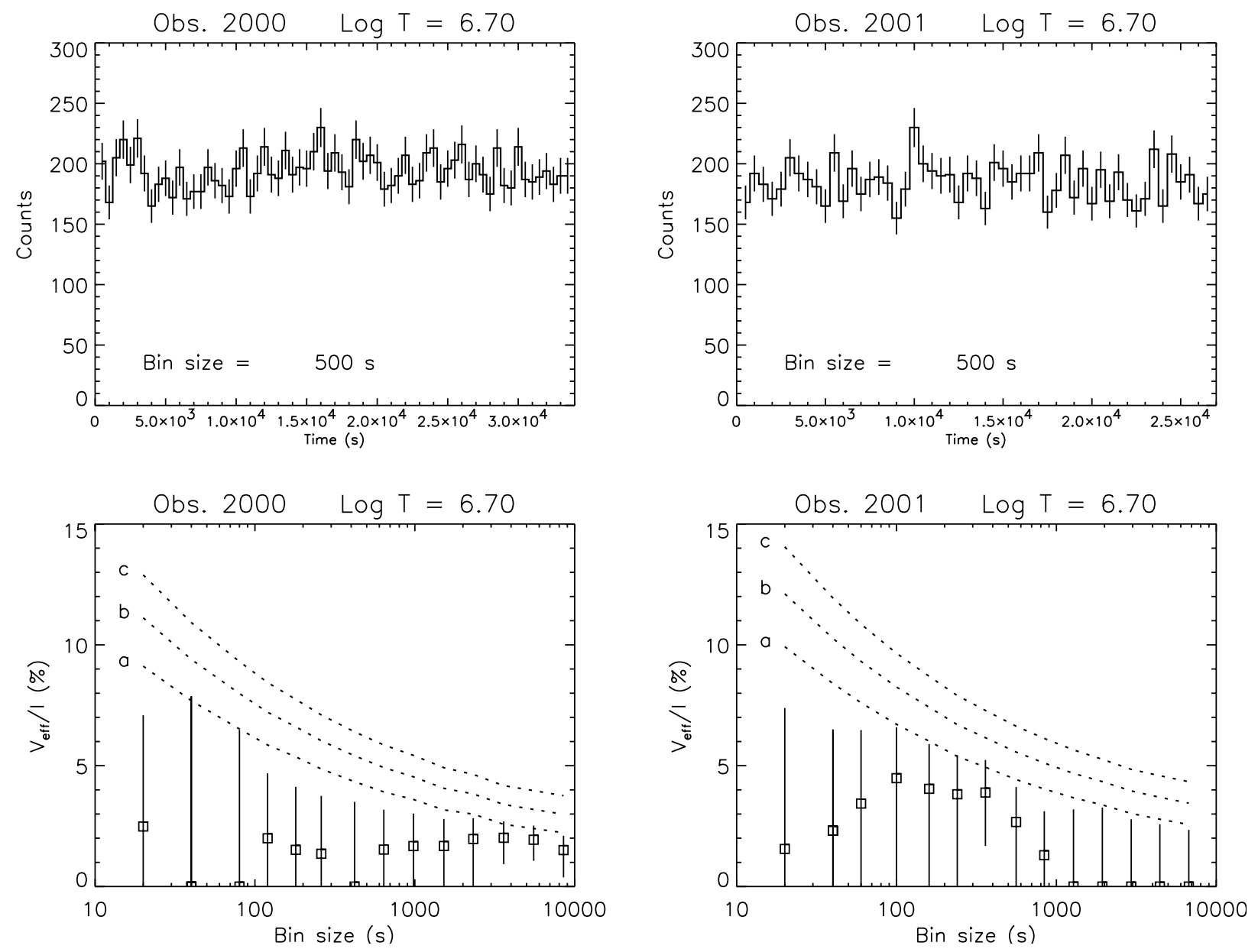

Fig. 10. Light curves and effective variability for the lines at $T_{\max }=10^{6.7} \mathrm{~K}$ for both the observations. The upper panels show the light curves generated collecting all the photons of selected lines with the same $T_{\max }$ (see Table 4). The lower panels show the values of effective variability $V_{\text {eff }} / I$ found for each bin size explored. Dotted curves correspond to the statistical confidence levels at $(a) 95.45 \%,(b) 99.73 \%$ and (c) $99.99 \%$ that the null hypothesis of constant emission is true.

\subsubsection{Variations over time scales of one year}

A significant information can be derived from the mean source count rate relative to each observation: the computed values $\left(2.726 \pm 0.011 \mathrm{cts} \mathrm{s}^{-1}\right.$ and $2.638 \pm 0.012 \mathrm{cts} \mathrm{s}^{-1}$ for the 2000 and 2001 observations respectively) differ by 5 standard deviations and show that during the first observation the coronal emission was $\sim 3 \%$ more intense than one year later.

We have then compared the line intensities measured in the two spectra: Fig. 8 shows the differences between the line fluxes versus $T_{\max }$, the temperature of peak emissivity. All these differences are within $3 \sigma$, but it is evident that the fluxes of the first observation are systematically larger than those of the second, in agreement with the finding based on the mean count rate. Moreover the differences tend to be larger for the lines with formation temperatures between $10^{6.5} \mathrm{~K}$ and $10^{7} \mathrm{~K}$. In order to improve the statistics we have summed all the fluxes from the lines with the same $T_{\max }$, and then we have compared the values obtained from the two observations (see Fig. 9); the maximum discrepancies are $4 \sigma$ and $3.4 \sigma$ for the groups of lines which forms at $\log T_{\max }=6.7$ and 6.9 respectively. Note that at these temperatures, which are near the peak of the emission measure distribution, we have the higher values for the summed line fluxes, that is to say the best sensitivity to the variability of the line emission.

\subsubsection{Short-term variability}

We have performed a detailed search of short-term variability using the phase-averaged $\chi^{2}$ method proposed by Collura et al. (1987). Following this procedure we have binned the photon arrival times in equal duration intervals, ranging from $50 \mathrm{~s}$ to $8000 \mathrm{~s}$; then we have compared each binned light curve with a constant one. Since the $\chi^{2}$ value depends on the bin size $b$ and the initial phase $\varphi$, i.e. $\chi^{2}=\chi^{2}(b, \varphi)$, according to Collura et al. we have evaluated for each bin size $b$ the phase-averaged $\overline{\chi^{2}}(b)$ value, and then we have compared these values with statistical confidence levels computed under the hypothesis of a constant source. With this procedure it is possible to test, in a statistically significant way, the presence of variability, and then, assuming a pulsed variability model, to evaluate the characteristic time scale $\tau$ and the fractional variability level $V_{\text {eff }} / I$ vs. the bin size $b$, where $V_{\text {eff }}$ is the amplitude of the variable component and $I$ is the average number of counts per bin (see Collura et al. 1987, for more details). 


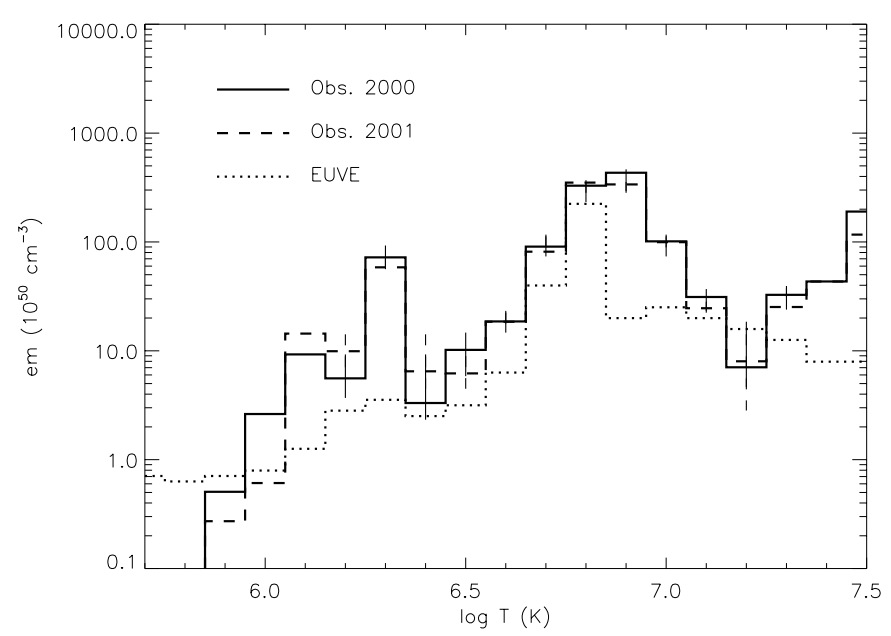

Fig. 11. Our emission measure distributions (solid for observation taken in 2000 and dashed for observation taken in 2001) and EUVE emission measure distribution (dotted) obtained by Dupree et al. (2003).

We have first applied this procedure to the arrival times of all the photons in each spectrum, finding that the emission is compatible with the hypothesis of a constant source, at high statistical confidence level. Note that the whole spectrum is the results of the emission by plasma characterized by a wide range of temperatures. In principle it is possible that the emission from small amounts of plasma, in narrow temperature ranges, may not be constant, and that this effect is lost analyzing the light curve of the whole spectrum. Therefore we have applied the method to individual lines, thus studying the variability of radiation emitted by the plasma with temperature close to $T_{\max }$ for each selected line.

For each line we have fixed a wavelength range $\Delta \lambda$ and we have extracted the photon arrival times in that range ${ }^{4}$. We have also analyzed together the arrival times of different lines with the same $T_{\max }$ value, in order to increase the photon counting statistics: in this way we were able to test for variability down to a sensitivity limit of 5-10\%. In Fig. 10 we show the light curve and the plot of the effective variability vs. the bin size $b$ for two representative cases. For each temperature, we have found that the emission is compatible with the hypothesis of a constant source. In Table 4 we report the results obtained including the effective variability upper limit at the $99.7 \%$ confidence level evaluated at a representative value of $b=10^{3} \mathrm{~s}$.

\section{Discussion}

\subsection{Variability}

We have found a marginal long-term variability from the total count rate, but also by comparing line fluxes measured one

\footnotetext{
4 It is worth noting that within each line spectral range it is not possible to distinguish the source emission in the spectral line from its continuum emission, and from the instrumental and sky background. This fact is not so important because, for all the lines we have considered, the contamination is a small fraction of the total number photons $(<25 \%)$. Moreover we have verified that the background taken on both sides of the spectrum was constant.
}

Table 5. Ascending and descending slope values for the emission measure distributions. The fitting has been performed over the $\log T$ intervals $[6.5,6.8]$ and $[6.9,7.2]$.

\begin{tabular}{ccc}
\hline \hline & $e m(T)$ Obs. 2000 & $e m(T)$ Obs. 2001 \\
\hline$\beta$ & $5.7 \pm 1.3$ & $6.1 \pm 0.9$ \\
$\alpha$ & $5.7 \pm 1.4$ & $5.6 \pm 1.1$ \\
\hline
\end{tabular}

year apart. Both these methods have shown that during the observation made in 2000 the coronal emission was $3 \%$ (at $5 \sigma$ level) higher than during the second one made in 2001. On the other hand, we have found overall compatibility between the emission measure distributions of the two observations, given the higher error bars in $\operatorname{em}(T)$. We have found that the largest differences between measured line counts occur for lines with $T_{\max }$ near the peak temperature of the emission measure distribution, which are also those with the highest signal to noise ratio.

The search of short-term variability inside each observation allows us to pose only upper limits on variable components, suggesting that the coronal structure of Capella is stable on time scales ranging from one minute to a few hours. It is reasonable to assume that the $\mathrm{X}$-ray coronal emission is produced by relatively stable structures. This is consistent with the fact that Capella does not show large flux variations.

\subsection{Emission measure distribution and coronal loop population}

Figure 11 shows the em $(T)$ derived from the two Chandra observations together with the em $(T)$ derived from $E U V E$ data by Dupree et al. (2003), based on an observation made in 2001. Our emission measure has a peak at $T_{j}=10^{6.9} \mathrm{~K}$ wider than that found by Dupree et al. Moreover, we observe a large difference at $T_{j}=10^{6.3} \mathrm{~K}$ between our $\operatorname{em}\left(T_{j}\right)$ and the one from $E U V E$ data. Dupree et al. have also obtained a minimum for $e m(T)$ at $T=10^{5.8} \mathrm{~K}$ which however is outside our temperature range.

Following the results of our variability analysis we can assume that the corona of Capella is made of stationary magnetic loops which are the largely dominant (or the only) source of the observed emission. From the physical conditions of the corona of Capella we infer that the pressure is approximately uniform inside these loops, because the pressure scale height, $H_{\mathrm{p}}$, is of the order of the stellar radius (and much larger than in the solar case), due to the high coronal temperature and to the low surface gravity $\left(g_{*} \sim 0.02 g_{\odot}\right)$ : already for $T=10^{6} \mathrm{~K}$ we obtain $H_{\mathrm{p}} \sim 10^{11} \mathrm{~cm}$, which is slightly smaller than the stellar radius $\left(R_{*} \sim 10^{12} \mathrm{~cm}\right)$, and for $T=10^{7} \mathrm{~K}$ we obtain $H_{\mathrm{p}} \sim 10^{12} \mathrm{~cm}$. Therefore we can argue that the pressure has to remain constant along any loop as large as the stellar radius or smaller. As we shall prove in Sect. 4.4, volume estimates based on the analysis of the He-like triplets suggest characteristic lengths ( $L \sim 10^{9}-10^{11} \mathrm{~cm}$ ) smaller than the stellar radius, confirming the hypothesis that the plasma in the coronal loops is isobaric. 
If we assume the loop model proposed by Rosner et al. (1978), we find that the shape of the emission measure distribution for a single loop does not depend on the size of the loop itself, but only on the loop maximum temperature $T_{\mathrm{p}}$ (Maggio $\&$ Peres 1996), and its functional form is $e m(T) \propto T^{\beta}$ for $T<T_{\mathrm{p}}$. We have used the approach proposed by Peres et al. (2001) who show that it is possible to infer information about the coronal structure starting from the consideration that the total $\mathrm{em}(T)$ of the whole corona is the sum of the emission measure distributions of individual loops. Under these assumptions Peres et al. have shown that the ascending slope of the $\mathrm{em}(T)$ of the whole corona is linked to the $\beta$ value, and the power-law index $-\alpha$ of the descending slope is linked to the distribution of the maximum temperatures of different classes of coronal loops. We have performed this analysis estimating the ascending and descending slopes of the Capella $\operatorname{em}(T)$ in the intervals: $\log T=6.5-6.8$ and $\log T=6.9-7.2$ respectively (Table 5).

The value $\beta=1.5$ corresponds to the hypothesis of constant loop cross-section and uniform heating, and Peres et al. (2001) have shown that the solar $\operatorname{em}(T)$ is well described by this model. The much larger values we have found for $\beta$ (Table 5) suggest two possibilities: 1) the heating is not uniform but higher near the loop footpoints, in this way the temperature profile along the loop is flatter, and therefore the emission measure distribution vs. $T$ is steeper than in the case of uniform heating, since the emission measure is concentrated in a narrower temperature range; 2) the loop cross-section increases with height, implying that there is more emission measure at higher temperatures; it is however hard to get such a steep $\operatorname{em}(T)$ even assuming a strong opening. Also the two effects may be both at work, since the two hypothesis are not mutually exclusive. The high values found for $\alpha$ show that almost all the loops have a $T_{\mathrm{p}}$ value equal to, or only slightly larger than, the peak value of the total emission measure distribution. In Fig. 12 we show the distribution $N f(T) e m\left(T_{\mathrm{p}}\right)$ (according to the notation of Peres et al. 2001) of loop maximum temperature, where $N$ is the total number of loops, $f(T)$ is the fraction of loops with maximum temperature $T_{\mathrm{p}}$, and $\operatorname{em}\left(T_{\mathrm{p}}\right)$ is the maximum value of the emission measure distribution of one loop with maximum temperature equal to $T_{\mathrm{p}}$. A consequence of our hypothesis is that the distribution evaluated does not contain a significant amount of loops with maximum temperature lower than the peak temperature $10^{6.8} \mathrm{~K}$, however this scenario has to be taken as just an approximation and loops with $T_{\mathrm{p}}<10^{6.8} \mathrm{~K}$ may indeed exist albeit having a marginal role in determining the whole corona $\operatorname{em}(T)$. We argue that these loops have to exist because the whole $\operatorname{em}(T)$ at temperatures smaller than the peak value is not well described by a simple power-law; these loops are responsible for the amount of emission measure observed near $10^{6.3} \mathrm{~K}$, but we cannot analyze the $e m(T)$ shape near this secondary peak because we have not enough information (in fact we can assign error bars to the emission measure distribution only for $T \geq 10^{6.2} \mathrm{~K}$ ). It is worth noting also that the emission measure distribution obtained by Dupree et al. (2003, Fig. 11) shows a bump near $T=10^{6.3} \mathrm{~K}$, though the amplitude is lower than in our case, confirming our guess about another class of loops with peak temperature of a few million degrees. Moreover, the existence of this loop class will be more evident

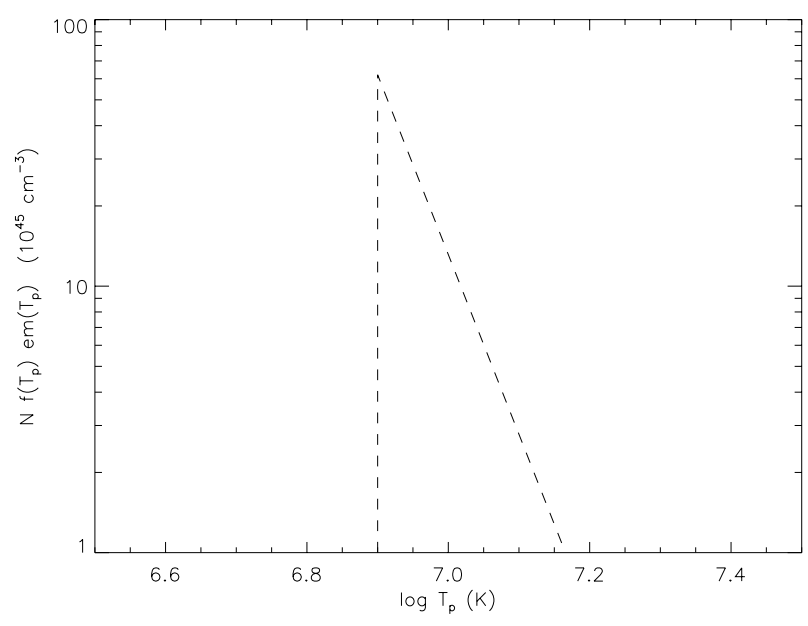

Fig. 12. Distribution of loops vs. their maximum temperature $T_{\mathrm{p}}$.

in the following analysis of the results obtained from the Helike triplets (see Sect. 4.4). Finally we note that we have also marginal indication about a third loop class needed to describe the hottest part of our $e m(T)$.

\subsection{Density and temperature values from He-like ions}

The density values that we have derived are compatible with those obtained by Brinkman et al. (2000), Canizares et al. (2000), Ness et al. (2001), Mewe et al. (2001), Phillips et al. (2001) and Audard et al. (2001). Our results show that the electron density $N_{\mathrm{e}}$ is higher in plasma regions with higher temperature. This finding is also supported by Dupree et al. (1993), they found densities ranging from $10^{11} \mathrm{~cm}^{-3}$ to $10^{13} \mathrm{~cm}^{-3}$ from the ratios of ultraviolet Fe XXI lines which form at $T \sim 10^{7} \mathrm{~K}$.

As already noted in Sect. 3.4 all the temperature values we have derived from the He-like ions are invariably lower than the corresponding temperature of peak emissivity $T_{\max }$; this finding is puzzling if we consider that, on the basis of the shape of the computed $\operatorname{em}(T)$, we would have expected that temperature values estimated from the O VII, Ne IX and Mg XI were higher than the corresponding $T_{\max }$ because there is a larger amount of emitting plasma at $T>T_{\max }$ than at lower $T$. As a check we have computed the temperature-sensitive $R_{\mathrm{T}}$ ratios (Sect. 3.4) with line fluxes synthesized from the emission measure distribution and found $R_{\mathrm{T}}$ lower than the observed ones, and the relevant temperature higher than $T_{\max }$.

A possible explanation of this result is that the CHIANTI V3.03 database does not include a sufficient number of dielectronic satellite lines, produced by a doubly excited state in a Li-like ion. If taken into account, these satellite lines yield to higher predicted $R_{\mathrm{T}}$ values, and a higher estimate for $T$ (Porquet et al. 2001). We stress that all the $R_{\mathrm{T}}$ values we have obtained are compatible with those reported by Brinkman et al. (2000), Canizares et al. (2000), Ness et al. (2001), Mewe et al. (2001), Phillips et al. (2001) and Audard et al. (2001), confirming the hypothesis that the observed behavior is linked to the atomic model adopted and not to instrumental effects or line fitting problems. 

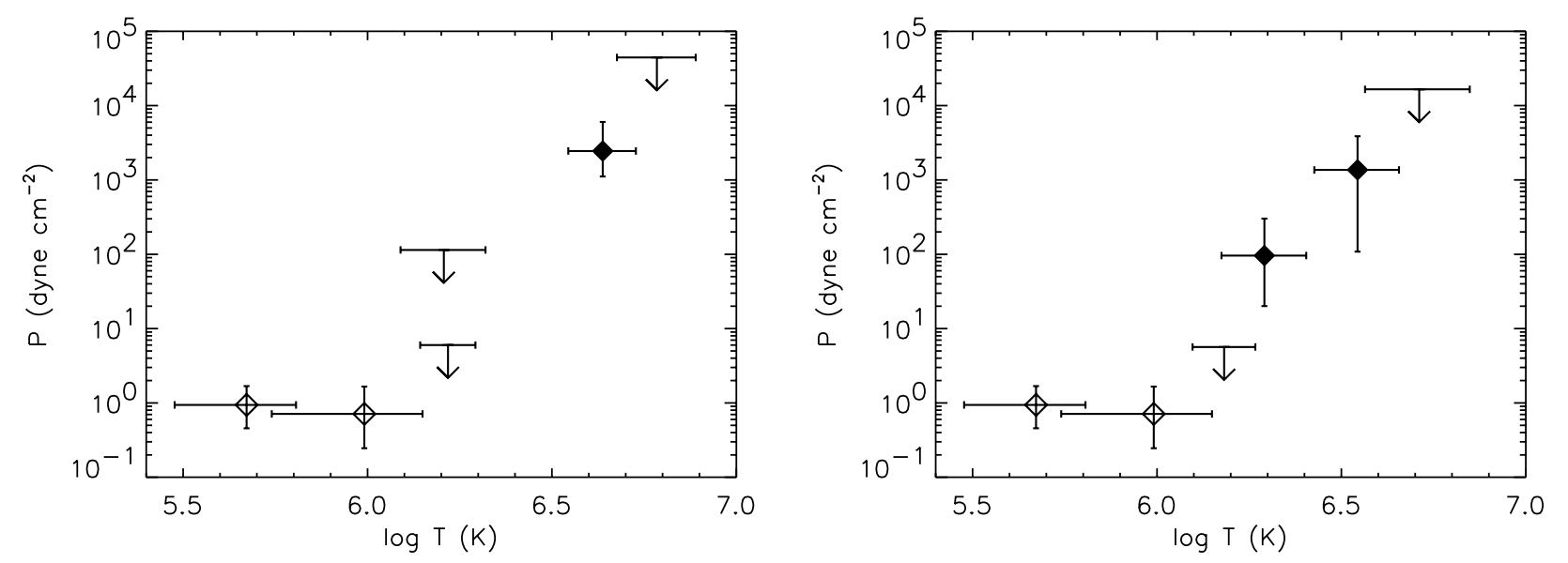

Fig. 13. Coronal plasma pressure vs. temperature for the two observations. The pressures are obtained by the density and temperature values evaluated from the analysis of He-like triplets. Filled diamonds and upper limits indicate the values determined by our analysis of O VII, Ne IX, Mg XI and Si XIII triplet, while open diamonds indicate pressures obtained by the C V and N VI analysis made by Ness et al. (2001).

Table 6. Volume of coronal plasma at different temperatures evaluated from density values from He-like triplet diagnostics.

\begin{tabular}{lcccccc}
\hline \hline \multicolumn{5}{c}{ Obs. 2000 } & \multicolumn{3}{c}{ Obs. 2001 } \\
\hline Ion & $\log T^{*}$ & $E M\left(T^{*}\right)\left(\mathrm{cm}^{-3}\right)$ & $V\left(\mathrm{~cm}^{3}\right)$ & $\log T^{*}$ & $E M\left(T^{*}\right)\left(\mathrm{cm}^{-3}\right)$ & $V\left(\mathrm{~cm}^{3}\right)$ \\
\hline O VII & 6.2 & $\sim 2 \times 10^{52}$ & $>2 \times 10^{32}$ & 6.2 & $\sim 2 \times 10^{52}$ & $>2 \times 10^{32}$ \\
Ne IX & 6.2 & $\sim 1 \times 10^{54}$ & $>3 \times 10^{31}$ & 6.3 & $\sim 4 \times 10^{53}$ & $\sim 1 \times 10^{31}$ \\
Mg XI & 6.6 & $\sim 2 \times 10^{53}$ & $\sim 5 \times 10^{28}$ & 6.5 & $\sim 4 \times 10^{53}$ & $\sim 2 \times 10^{29}$ \\
Si XIII & 6.8 & $\sim 2 \times 10^{53}$ & $>5 \times 10^{26}$ & 6.7 & $\sim 3 \times 10^{53}$ & $>4 \times 10^{27}$ \\
\hline
\end{tabular}

\subsection{Volume and pressure from He-like ions}

The values of $T$ and $N_{\mathrm{e}}$ obtained from the study of He-like ions allow us to estimate the effective volume and the average pressure of the region where these lines are formed. To determine the volume $V$ we have evaluated the emission measure em $\left(T^{*}\right)$ of the whole region using the resonance line emissivity at the temperature $T^{*}$ derived from the $R_{\mathrm{T}}$ ratio. Then, the volume is estimated as $V=e m\left(T^{*}\right) / N_{\mathrm{e}}^{2}$, and the values obtained are shown in Table 6 . We stress that this procedure assumes that all the plasma contributing to the He-like emission is concentrated at the single temperature $T^{*}$. Phillips et al. (2001) derived from the $\mathrm{O}$ VII line triplet an estimate of the volume of $\sim 4 \times 10^{31} \mathrm{~cm}^{3}$ (with an uncertainty range $1 \times 10^{31}-2 \times 10^{32} \mathrm{~cm}^{3}$ ), while Behar et al. (2001) have reported a volume of $\sim 10^{29} \mathrm{~cm}^{3}$ at $T=10^{6.8} \mathrm{~K}$, derived from iron lines: both these estimates are consistent with our findings.

In order to derive the size of individual loops from the above estimates of the effective plasma volumes we must consider two effects: each loop is characterized by a large temperature range, while the volumes are based on emission lines which form over a restricted temperature range; moreover the corona of Capella is most likely made of a large number of loops which contribute to the line emission and therefore to the effective volume estimates. We guess that the second effect dominates the first one and therefore the effective volume estimates represent upper limits to individual loop volumes. Hence the characteristic sizes of the coronal structures $\left(L \sim V^{1 / 3}\right)$, supported also by the results from Phillips et al. and Behar et al., are $\sim 10^{11} \mathrm{~cm}$ for plasma at $T \sim 10^{6} \mathrm{~K}$ and $\sim 10^{9} \mathrm{~cm}$ for $T \sim 10^{7} \mathrm{~K}$. The comparison between these characteristic lengths and the pressure scale height confirms the hypothesis of isobaric loops.

We have noted above (Sect. 3.4) that the temperatures $T^{*}$ derived from the observed $R_{\mathrm{T}}$ may be slightly underestimated, implying that the volumes obtained using these temperatures may be imprecise. To quantify this effect we have also evaluated the volume values using, instead of $T^{*}$, the effective temperatures obtained from the $R_{\mathrm{T}}$ ratio evaluated by the predicted line fluxes: the volumes obtained are smaller by a factor $2-5$, and therefore the hypothesis of constant pressure loops are even more confirmed.

As a further step it is possible to estimate the average pressure $P$, relative to the region where the triplets are formed, from the value of $T^{*}$ and $N_{\mathrm{e}}$. In Fig. 13 we show plots of $P$ vs. $T^{*}$ for the two observations. From this plot we deduce that the plasma present in the corona of Capella, confined in magnetic loops, is characterized by very different pressure values: in particular, we have found that the higher the temperature, the larger the pressure values. We note that this result is not in conflict with the hypothesis of constant pressure loops: in fact, it may happen that the pressure is very different from one loop to another, but remains constant inside each loop. We can state that different loop classes with different pressures characterize the corona of Capella, and loops with higher pressures have also higher values of maximum temperature $T_{\mathrm{p}}$. Recalling the shape of the emission measure distribution (Fig. 11) and the analysis made in Sect. 4.2, we can tentatively identify the high-pressure loops with the dominant class of coronal structures having 
$T_{\mathrm{p}} \sim 10^{6.8} \mathrm{~K}$, and the low-pressure loops with the structures determining the secondary peak in the $\operatorname{em}(T)$ at $T \sim 10^{6.3} \mathrm{~K}$. In order to test qualitatively this coronal model, we plan to perform detailed simulations, whose results will be reported in a subsequent paper.

\section{Conclusions}

In this work we have analyzed two LETGS observations of Capella for which we have identified and measured the strongest emission lines. Starting from the reconstructed emission measure distribution we have obtained information about the structure of individual loops and we have derived the coronal loop distribution vs. peak temperature. The corona of Capella appears to be composed mainly of stable coronal structures in isobaric conditions, with characteristic sizes most likely smaller than the stellar radius, but such structures appear to have plasma emission measure distributions vs. temperature steeper than in the case of solar-like coronal structures. Our analysis indicates that different loop classes are needed to describe the corona of Capella: the dominant class is composed of loops having peak temperatures $T_{P} \sim 10^{6.8} \mathrm{~K}$, but we have also found evidence of at least another class of loops having $T_{P} \leq 10^{6.3} \mathrm{~K}$. In fact, the average pressure, estimated from the analysis of the He-like triplets, is significantly lower at $T \leq 1.5 \times 10^{6} \mathrm{~K}$ than at higher temperatures: since the loops are isobaric, this result indicates that the hotter loops must have plasma pressure higher than cooler loops.

Ayres et al. (1998), studying the broadening of UV lines with $T_{\max } \sim 10^{4.5}-10^{5.5} \mathrm{~K}$ in the Capella spectra taken with the Goddard High-Resolution Spectrometer of the Hubble Space Telescope, have suggested the existence of transition zone structures, having sizes comparable to the stellar radius, in which the plasma pressure, evaluated from the densitysensitive O IV line ratios, is $P \sim 0.2$ dyne $^{-2}$ at $T \sim 10^{5.2} \mathrm{~K}$ (Linsky et al. 1995). This value is compatible with the pressure estimated at $T \sim 10^{6} \mathrm{~K}$ within statistical uncertainties. However we argue that if these structures are the footpoints of the cool coronal loops, it is difficult to reconcile their extent with the hypothesis of isobaricity. On the other hand, the structures evidenced by Ayres et al. are certainly not in pressure equilibrium with the coronal loops which explain the bulk of the X-ray (and EUV) emission from Capella.

The next step in our investigation will be to derive a more detailed model of the corona which will help us to interpret all the observational evidences presented in this paper.

Acknowledgements. We thank V. Kashyap and J. Drake for allowing us to use their software for the emission measure reconstruction. We also thank J. Sanz-Forcada who provided us with the emission measure distribution of Capella derived from a recent EUVE observation. Finally we acknowledge partial support for this work by Agenzia Spaziale Italiana and Ministero dell'Istruzione, dell'Università e della Ricerca.

\section{References}

Audard, M., Behar, E., Güdel, M., et al. 2001, A\&A, 365, L329

Ayres, T. R., Brown, A., Osten, R. A., et al. 2001, ApJ, 549, 554

Ayres, T. R., Simon, T., Stern, R. A., et al. 1998, ApJ, 496, 428

Bauer, F., \& Bregman, J. N. 1996, ApJ, 457, 382

Behar, E., Cottam, J., \& Kahn, S. M. 2001, ApJ, 548, 966

Brickhouse, N. S., Dupree, A. K., Edgar, R. J., et al. 2000, ApJ, 530, 387

Brinkman, A. C., Gunsing, C. J. T., Kaastra, J. S., et al. 2000, ApJ, 530, L111

Brown, G. V., Beiersdorfer, P., Liedahl, D. A., et al. 2002, ApJS, 140, 589

Canizares, C. R., Huenemoerder, D. P., Davis, D. S., et al. 2000, ApJ, 539, L41

Collura, A., Maggio, A., Sciortino, S., et al. 1987, ApJ, 315, 340

Dupree, A. K., Brickhouse, N. S., Doschek, G. A., Green, J. C., \& Raymond, J. C. 1993, ApJ, 418, L41

Dupree, A. K., Brickhouse, N. S., \& Sanz-Forcada, J. 1999, Bull. Am. Astron. Soc., 31, 1483

Dupree, A. K., Brickhouse, N. S., \& Sanz-Forcada, J. 2003, in preparation

Favata, F., Mewe, R., Brickhouse, N. S., et al. 1997, A\&A, 324, L37

Gabriel, A. H., \& Jordan, C. 1969, MNRAS, 145, 241

Grevesse, N., Noels, A., \& Sauval, A. J. 1992, in Coronal Streamers, Coronal Loops, and Coronal and Solar Wind Composition, in ESA Proceedings of the First SOHO Workshop, 305

Hummel, C. A., Armstrong, J. T., Quirrenbach, A., et al. 1994, AJ, 107,1859

Johnson, O., Drake, J. J., Kashyap, V., et al. 2002, ApJ, 565, L97

Kashyap, V., \& Drake, J. J. 1998, ApJ, 503, 450

Kashyap, V. L., \& Drake, J. J. 2000, AAS/High Energy Astrophysics Division

Keenan, F. P., Kingston, A. E., \& Tayal, S. S. 1984, Sol. Phys., 92, 75

Linsky, J. L., Brown, A., Gayley, K., et al. 1993, ApJ, 402, 694

Linsky, J. L., Wood, B. E., Brown, A., \& Osten, R. A. 1998, ApJ, 492, 767

Linsky, J. L., Wood, B. E., Judge, P., et al. 1995, ApJ, 442, 381

Maggio, A., \& Peres, G. 1996, A\&A, 306, 563

Mazzotta, P., Mazzitelli, G., Colafrancesco, S., \& Vittorio, N. 1998, A\&AS, 133, 403

Mewe, R., Raassen, A. J. J., Drake, J. J., et al. 2001, A\&A, 368, 888

Ness, J.-U., Mewe, R., Schmitt, J. H. M. M., et al. 2001, A\&A, 367, 282

Ness, J.-U., Schmitt, J. H. M. M., Burwitz, V., et al. 2002, A\&A, 394, 911

Peres, G., Orlando, S., Reale, F., \& Rosner, R. 2001, ApJ, 563, 1045

Perryman, M. A. C., Lindegren, L., Kovalevsky, J., et al. 1997, A\&A, 323, L49

Phillips, K. J. H., Mathioudakis, M., Huenemoerder, D. P., et al. 2001, MNRAS, 325, 1500

Porquet, D., \& Dubau, J. 2000, A\&AS, 143, 495

Porquet, D., Mewe, R., Dubau, J., Raassen, A. J. J., \& Kaastra, J. S. 2001, A\&A, 376, 1113

Pradhan, A. K. 1982, ApJ, 263, 477

Rosner, R., Tucker, W. H., \& Vaiana, G. S. 1978, ApJ, 220, 643

Schrijver, C. J., Mewe, R., van den Oord, G. H. J., \& Kaastra, J. S. 1995, A\&A, 302, 438

Young, P. R., Dupree, A. K., Wood, B. E., et al. 2001, ApJ, 555, L121 